\title{
Composition of Safety Constraints For Fixed-Wing Collision Avoidance Amidst Limited Communications
}

\author{
Eric Squires* and Pietro Pierpaoli ${ }^{\dagger}$ \\ Georgia Institute of Technology, Atlanta, GA 30318 \\ Rohit Konda \\ University of California Santa Barbara, Santa Barbara, California 93106 \\ Samuel Coogan ${ }^{\S}$ \\ Georgia Institute of Technology, Atlanta, GA 30318 \\ Magnus Egerstedt ${ }^{\mathbb{I I}}$ \\ University of California, Irvine 92697
}

\begin{abstract}
This paper considers how to ensure that a system of fixed wing Unmanned Aerial Vehicles (UAVs) can avoid collisions. To do so we develop a novel method for creating a barrier function, which is similar to a Lyapunov function and can be used to ensure that a system can stay safe for all future times. After introducing the general approach, it is shown how to ensure that collision avoidance for two vehicles can be guaranteed for all future times. The construction is then extended to the case of arbitrarily many vehicles by addressing how to satisfy multiple safety objectives simultaneously. We do this while ensuring output actuator commands are within specified limits. Because this formulation requires communication of control values and may therefore reduce throughput of other important messages, we then show how to reformulate the solution without this significant communication overhead while still ensuring safety is maintained and actuator limits are respected. We validate the theoretical developments of this paper in the simulator SCRIMMAGE with a simulation of 20 UAVs that maintain safe distances from each other even though their nominal paths would otherwise cause a collision.
\end{abstract}

\section{Introduction}

A s low-cost, unmanned aerial vehicles (UAVs) find civilian uses, the low-altitude airspace is increasingly congested, leading to large-scale UAV operation limitations including concerns for privacy, the environment, national security, and safe-flight validation [1]. A key challenge for safe-flight validation in congested environments is ensuring

\footnotetext{
*Research Engineer, Aerospace, Transportation and Advanced Systems Laboratory of the Georgia Tech Research Institute; eric.squires@gtri.gatech.edu.

†Postdoc, Electrical and Computer Engineering; pietro.pierpaoli@gatech.edu.

$\$ \mathrm{PhD}$ student, Electrical and Computer Engineering.

$\S$ Assistant Professor, School of Electrical and Computer Engineering as well as the School of Civil and Environmental Engineering; sam.coogan@gatech.edu.

II Dean, Samueli School of Engineering, magnus@uci.edu
} 
collision avoidance while enabling vehicles to accomplish their designed missions. Thus, in this paper we propose an algorithm that minimally alters a vehicle's nominal control input while still ensuring safe operations.

A variety of approaches to fixed-wing collision avoidance have been proposed. Partially observable Markov decision processes are used in [2, 3] to achieve safe flight distances. The dynamic window approach, originally introduced in [4] for static obstacles and adapted to moving obstacles in [5], uses circular arcs for trajectories and limits the set of allowable velocities to enable a quick optimization of the control input. In [6], the authors develop a first-order look-ahead algorithm that can be applied to vehicles with unicycle dynamics in a decentralized way while guaranteeing that collisions amongst $k$ vehicles are avoided. Potential functions [7, 8] have also been applied to fixed-wing collision avoidance, where it can be shown that vehicles can safely avoid each other even when their sensing range is limited. Similarly, [9] discusses how to combine potential functions with trajectory goals into a navigation function in order to provide criteria under which collision avoidance can be guaranteed. Navigation functions have also been combined with Model Predictive Control (MPC) by making inter-agent distance requirements implicit in the cost function [10]. MPC has additionally been applied to UAV collision avoidance for vehicles with limited sensing [11] and communication constraints [12]. While MPC provides a flexible framework for distributed collision avoidance, its limited horizon can make safety guarantees difficult. In a more general case, the optimal control formulation in [13] allows for collision avoidance guarantees, but it is computationally intensive as it requires numerically solving the Hamilton-Jacobi-Bellman equations over an infinite horizon.

Trajectory generation was analyzed in [14] where a nonlinear program is developed to find a safe reference trajectory constructed from polynomials. In [15] and [16], the authors discuss trajectory generation using a RRT with dynamics constraints provided by Dubins paths and a waypoint generation algorithm, respectively. Reference governors [17], where the input reference signal for a nominal closed loop controller is overridden in order to ensure that safety and performance constraints are maintained, have also been applied to collision avoidance in [18]. In [18] the authors show how to ensure collision avoidance for a distributed set of linear systems via a sequential mixed-integer programming optimization. The approach considers a finite horizon in the optimization because it is shown that a constant reference can then keep the system safe after that point. Reference governors are similar to the approach of this paper in that given a nominal controller the approach seeks a minimal adjustment in order to improve safety characteristics. However, they differ in how the minimal adjustment occurs. A reference governors adjusts the setpoint that a nominal system is designed to achieve. On the other hand, the approach of this paper does not require a reference input to the nominal system and instead allows a nominal controller to calculate a control input as it normally would. The approach of this paper then minimally adjusts the control input to ensure safety. Finally, in [19], the authors also consider a trajectory based approach to avoid static obstacles. Similar to evasive maneuvers, traffic rules [20, 21] are a method for encoding hybrid behaviors that can include collision avoidance trajectories. In [20], the authors show that a two vehicle system with limited sensing range can avoid collisions while reaching position goals. While in general this may result in 
conservative behaviors, they demonstrate in simulation that the decentralized algorithm continues to allow vehicles to reach their target configuration while avoiding collisions for as many as 70 vehicles. Reactive methods are useful because they can often be calculated online while evasive maneuvers benefit from a lookahead into the future. In this paper we leverage the merits of both approaches within the framework of control barrier functions.

Motivated by the importance of formal guarantees of collision avoidance that are computationally feasible and minimally invasive we discuss in this paper how to apply barrier functions (e.g., [22], [23]) to the UAV collision avoidance problem, where the system is subject to actuator constraints, nonlinear dynamics, and nonlinear safety constraints. Barrier functions are similar to Lyapunov functions and allow for guarantees that a system will stay safe (i.e., vehicles will maintain safe distances from each other) for all future times. Further, under some assumptions detailed in Section $\Pi$ a Quadratic Program (QP) can be used to calculate a safe control input implied by a barrier function so that the calculation can be done online [23]. Given such safety guarantees, barrier functions have been applied to a set of problems including collision avoidance for autonomous agents $([24,25])$, bipedal robots $([26,27])$, adaptive cruise control and lane following ([23, 28,-30]), and in mobile communication networks [31].

However, barrier functions rely on being able to find a function for safety set invariance to be guaranteed. For systems like a fixed wing UAV with actuator constraints, nonlinear dynamics, and nonlinear safety constraints, generating such a function can be difficult. In this respect they are similar to Lyapunov functions. They provide guarantees when a system designer can find appropriate functions but they may be difficult to construct.

Nevertheless, there are a variety of approaches to finding a barrier function given a system and safety constraints. One approach discussed for instance in $([22,28,32,33])$, uses a sum of squares decomposition [34]. In this approach an initially conservative estimate for a barrier function is found and the associated safe set is iteratively enlarged. Iterative approaches have also been developed when the system has relative degree greater than one. The conditions for calculating a safe control input for higher order systems are given in [35]. In [27], a backstepping approach is developed that ensures a control barrier function can be constructed and a similar approach is discussed in [36]. The approach discussed in this paper is most similar to [37] where a barrier function is formulated by calculating the distance to a backup set after applying a backup controller. In this paper we develop an alternative approach that does not require the specification of a backup set.

System-specific arguments have also been applied to the development of a barrier function. For instance, geometric insights are exploited in [26], where the authors develop a barrier function for precise foot placement by ensuring that the foot is within the intersection of two circles. Similarly, in [24, 25], the authors develop a barrier function that ensures a circle and ellipsoid, respectively, around each robot will not overlap in order to ensure there will be no collisions for double integrator and quadrotor robots, respectively. Barrier functions have also been developed for unicycle dynamics in [30], where the dynamics are simplified by considering a point slightly in front of the vehicle.

Previous work on barrier functions has shown how, given the current state, a safe control input can be selected to 
ensure the system is safe for all times. In this paper, we also ensure system safety but do so by integrating the dynamics into the future using a known evasive maneuver that is always available to keep the system safe. In this respect the system is more predictable since it is known that a particular control input will be safe. Further, we ensure that actuator limits are respected which is a significant constraint in the case of UAVs where the system has non-zero minimum velocity.

Aside from ensuring a barrier function constraint can be satisfied given actuator limitations, UAV collision avoidance also motivates the consideration of multiple safety constraints that must be satisfied at all times. In particular, because collision avoidance can be viewed as a constraint for each pairwise combination of vehicles [31, 38], we briefly review how barrier functions have been applied to systems with multiple constraints. A contract-based approach is presented in [28]. A sum of squares decomposition is presented in [33] where additional safety constraints map to additional constraints in the optimization problem. In [36], necessary and sufficient conditions are given for the existence of a control input that satisfies multiple barrier function constraints. The approach generalizes to high order and time-varying systems but requires that actuator constraints be unbounded. Barrier function composition has also been addressed in [28, 31, 38]. In [28], the authors partition the state space into regions for which a single barrier function is active in each component of the partition. In [31] and [38] non-smooth barrier functions are discussed, where the result allows for combining barrier functions using boolean primitives. One drawback of the boolean composition approaches is that it is not guaranteed that the composition of barrier functions will result in a barrier function.

The high level contribution of the paper is a method for constructing a barrier function given a safety constraint and system dynamics. In particular, after a safety engineer specifies an evasive maneuver we then show how to construct a barrier function. This paper makes the following technical contributions. First, it generalizes a method discussed in [24, 25] for constructing a barrier function that can be used to make safety guarantees for a system. Second, it examines how to ensure that multiple safety constraints can be satisfied simultaneously when using this constructive method. Third, it presents an algorithm for ensuring safety in the context of multi-agent systems that does not require communication of low level actuator commands. Fourth, it shows how to apply the above theory to a scenario involving fixed wing UAVs where vehicles must ensure minimum separation distances are maintained at all times. This paper expands on the conference version [39] which did not consider multiple constraints and did not consider limited communications. Finally, this paper expands on the simulation study presented in [39] by considering a scenario with 20 vehicles to demonstrate that all pairwise distances between vehicles can be kept above a minimum safety distance throughout a scenario.

This paper is organized as follows. Section [I] discusses background information for barrier functions. Section III] discusses a general method for constructing a barrier function and shows how to apply it to fixed wing collision avoidance. Section [V generalizes the results of Section $\amalg$ by showing how to satisfy multiple constraints simultaneously. Section $\mathrm{V}$ relaxes the amount of information required to share between vehicles while still guaranteeing safety. Section[V] presents 
a simulation verification of the approach. Section VII concludes.

\section{BARRIER FUNCTIONS BACKGROUND}

We summarize the necessary background for barrier functions here. See [23] for further discussion. Consider a control affine system

$$
\dot{x}=f(x)+g(x) u
$$

where $f$ and $g$ are locally Lipschitz functions, $x \in \mathbb{R}^{n}, u \in U \subset \mathbb{R}^{m}$, and solutions are forward complete, meaning the system has a unique solution for all time $t \geq 0$ given a starting condition $x(0)$.

We expand this formulation to a set of $k$ vehicles by considering each vehicle's state $x_{i}$ and dynamics $\dot{x}_{i}=$ $f_{i}\left(x_{i}\right)+g_{i}\left(x_{i}\right) u_{i}$ where $x_{i} \in \mathbb{R}^{n_{i}}, u_{i} \in U_{i} \subseteq \mathbb{R}^{m_{i}}$ and $i \in\{1, \ldots, k\}$. The overall state for the system is described by $x=\left[\begin{array}{llll}x_{1}^{T} & x_{2}^{T} & \cdots & x_{k}^{T}\end{array}\right]^{T} \in \mathbb{R}^{n}$ where $n=\sum_{i=1}^{k} n_{i}$ and $u=\left[\begin{array}{llll}u_{1}^{T} & u_{2}^{T} & \cdots & u_{k}^{T}\end{array}\right]^{T} \in U_{1} \times U_{2} \times \cdots \times U_{k}=U \subset \mathbb{R}^{m}$, where $m=m_{1}+\cdots+m_{k}$. In this case, (1) can be represented as

$$
\dot{x}=\left[\begin{array}{c}
f_{1}\left(x_{1}\right) \\
f_{2}\left(x_{2}\right) \\
\vdots \\
f_{k}\left(x_{k}\right)
\end{array}\right]+\left[\begin{array}{cccc}
g_{1}\left(x_{1}\right) & 0 & \cdots & 0 \\
0 & g_{2}\left(x_{2}\right) & \ldots & 0 \\
\vdots & \vdots & \ddots & \vdots \\
0 & 0 & \cdots & g_{k}\left(x_{k}\right)
\end{array}\right]\left[\begin{array}{c}
u_{1} \\
u_{2} \\
\vdots \\
u_{k}
\end{array}\right] .
$$

We model the state and control input to the single vehicle by $x_{i}=\left[\begin{array}{llll}p_{i, x} & p_{i, y} & \theta_{i} & p_{i, z}\end{array}\right]^{T}$ and $u_{i}=\left[\begin{array}{lll}v_{i} & \omega_{i} & \zeta_{i}\end{array}\right]^{T}$, respectively. Further, we assume in this paper that the vehicles experience small bank and pitch angles so that single vehicle dynamics can be approximated by

$$
\dot{x}_{i}=\left[\begin{array}{ccc}
\cos \left(\theta_{i}\right) & 0 & 0 \\
\sin \left(\theta_{i}\right) & 0 & 0 \\
0 & 1 & 0 \\
0 & 0 & 1
\end{array}\right]\left[\begin{array}{c}
v_{i} \\
\omega_{i} \\
\zeta_{i}
\end{array}\right]
$$

where the sets of bounded control inputs are $v_{i} \in\left[v_{\min }, v_{\max }\right]$ with $v_{\min }>0,\left|\omega_{i}\right| \leq \omega_{\max },\left|\zeta_{i}\right| \leq \zeta_{\max }$. Similar approaches to modeling fixed wing aircraft can be found in [6, 8-10, 20, 21]. We also assume that each vehicle can sense the state of every other vehicle.

Suppose the set of desired configurations is described by the superlevel set of an output function $h: \mathbb{R}^{n} \rightarrow \mathbb{R}$ defined 
on an open set $\mathcal{D} \subseteq \mathbb{R}^{n}$. The superlevel set of $h$ is then called the safe set and is defined as

$$
C=\{x \in \mathcal{D}: h(x) \geq 0\}
$$

The objective is now to establish the condition under which the state system belongs to $C$ for all times.

Definition 1. [23] Given a set $C \subset \mathbb{R}^{n}$ defined in 3 for a continuously differentiable function $h: \mathbb{R}^{n} \rightarrow \mathbb{R}$, the function $h$ is called a zeroing control barrier function $(Z C B F)$ defined on an open set $\mathcal{D}$ with $C \subset \mathcal{D} \subset \mathbb{R}^{n}$, if there exists a Lipschitz continuous extended class $\mathcal{K}$ function $\alpha$ such that

$$
\sup _{u \in U}\left[L_{f} h(x)+L_{g} h(x) u+\alpha(h(x))\right] \geq 0, \forall x \in \mathcal{D} .
$$

In the above definition $L_{f} h(x)=\frac{\partial h(x)}{\partial x} f(x)$ and $L_{g} h(x)=\frac{\partial h(x)}{\partial x} g(x)$ denote the Lie derivatives. From Definition 1 . it follows that the admissible control space is defined as

$$
K(x)=\left\{u \in U: L_{f} h(x)+L_{g} h(x) u+\alpha(h(x)) \geq 0\right\}
$$

Theorem 1. [23] Given a set $C \subset \mathbb{R}^{n}$ defined in (3) for a continuously differentiable function $h$, if $h$ is a ZCBF on $\mathcal{D}$, then any Lipschitz continuous controller $u: \mathcal{D} \rightarrow U$ such that $u(x) \in K(x)$ will render the set $C$ forward invariant.

In [23] it is also shown how to calculate $u(x) \in K(x)$ using a Quadratic Program (QP) to support fast, online calculations. In particular, assume there is some nominal control input $\hat{u} \in \mathbb{R}^{m}$ available that is designed to achieve some performance goal (e.g., path-following) that has not necessarily been designed to satisfy safety constraints. Additionally, we assume $U$ can be expressed as the set of all $u$ satisfying the linear inequality $A u \geq b$. A safe control input can then be calculated using the following Quadratic Program (QP)

$$
\begin{aligned}
u^{*}=\min _{u \in \mathbb{R}^{m}} & \frac{1}{2}\|u-\hat{u}\|^{2} \\
\text { s.t. } \quad & L_{f} h(x)+L_{g} h(x) u+\alpha(h(x)) \geq 0 \\
& A u \geq b .
\end{aligned}
$$

Note that by property (4, when $h$ is a ZCBF, (6) is guaranteed to be feasible when $x \in \mathcal{D}$. 


\section{BARRIER FUNCTION CONSTRUCTION}

In general, in order to apply the barrier function framework discussed in the previous section, one needs to define an appropriate barrier function $h(x)$ representative of the collision avoidance constraints the UAVs must satisfy. To this end, as shown in the following example, the design of a suitable barrier function for fixed wing vehicles is not trivial.

\section{A. Motivating Example}

In this section we discuss some difficulties with applying barrier functions to the fixed-wing collision avoidance problem via a concrete example. Consider a candidate ZCBF, $h$, that encodes a collision avoidance safety constraint in a system of two vehicles with state $x=\left[\begin{array}{ll}x_{1}^{T} & x_{2}^{T}\end{array}\right]^{T}$ and

$$
h(x)=d_{1,2}(x)-D_{s}^{2}
$$

where

$$
d_{1,2}(x)=\left(p_{1, x}-p_{2, x}\right)^{2}+\left(p_{1, y}-p_{2, y}\right)^{2}+\left(p_{1, z}-p_{2, z}\right)^{2}
$$

is the squared distance between vehicles 1 and 2 and $D_{s}$ is a positive minimum safety distance. To show why $h$ defined in (7) is not a ZCBF, we present an example where, even though the configuration of the aircraft is safe since $x \in C$, $h(x)$ does not satisfy constraint 44 . Let $x_{1}=\left[\begin{array}{lllll}-D_{s} / 2 & 0 & 0 & \epsilon\end{array}\right]^{T}$ and $x_{2}=\left[\begin{array}{llll}D_{s} / 2 & 0 & \pi & -\epsilon\end{array}\right]^{T}$ for some $\epsilon \geq 0$. First, we note that for $x=\left[\begin{array}{ll}x_{1}^{T} & x_{2}^{T}\end{array}\right]^{T} \in C, h(x) \geq 0$. Further,

$$
\begin{aligned}
\sup _{u \in U}\left[L_{f} h(x)+L_{g} h(x) u+\alpha(h(x))\right]=\sup _{u \in U}\left[2\left(p_{1, x}-p_{2, x}\right)\left(v_{1} \cos \theta_{1}-v_{2} \cos \theta_{2}\right)\right. \\
+2\left(p_{1, y}-p_{2, y}\right)\left(v_{1} \sin \theta_{1}-v_{2} \sin \theta_{2}\right) \\
\left.+2\left(p_{1, z}-p_{2, z}\right)\left(\zeta_{1}-\zeta_{2}\right)\right] \\
=\sup _{u \in U}\left[-2 D_{s}\left(v_{1}+v_{2}\right)+2 \epsilon\left(\zeta_{1}-\zeta_{2}\right)\right] \\
=-4 D_{s} v_{\text {min }}+2 \epsilon \zeta_{\text {max }} .
\end{aligned}
$$

Since $v_{\min }>0$ and $D_{s}>0$, if the two vehicles' initial positions satisfy $0 \leq \epsilon<2 D_{s} v_{\min } / \zeta_{\max }$ we observe that the quantity above does not satisfy constraint (4), i.e., $\sup _{u \in U}\left[L_{f} h(x)+L_{g} h(x) u+\alpha(h(x))\right]<0$. Therefore, we conclude that $h(x)$ defined in (7) is not a ZCBF. The problem with this candidate ZCBF is that it does not account for the fact that by the time the vehicles are close to colliding, it may be too late to avoid each other due to the limited turning radius and positive minimum velocity. 


\section{B. Constructing a Barrier Function via Evading Maneuvers}

In order to overcome the difficulties demonstrated in the example of Section III.A, we introduce a method to systematically construct a ZCBF from a safety constraint. Let $\rho: \mathcal{D} \rightarrow \mathbb{R}$ be a safety function that represents the safety objective we want to satisfy at all times so that $\rho(x) \geq 0$ indicates that the system is safe. In the example from Section III.A for vehicles $i$ and $j$,

$$
\rho(x)=d_{i, j}(x)-D_{s}^{2}
$$

Second, let $\gamma: \mathcal{D} \rightarrow U$ be a nominal evading maneuver. Section [III.C discusses specific examples of $\gamma$ for the UAV collision avoidance problem. For now, assuming $\gamma$ has been selected, let

$$
h(x ; \rho, \gamma)=\inf _{\tau \in[0, \infty)} \rho(\hat{x}(\tau)),
$$

be a candidate ZCBF where $\hat{x}$ and $\dot{\hat{x}}$ are given by

$$
\begin{gathered}
\hat{x}(\tau)=x+\int_{0}^{\tau} \dot{\hat{x}}(\eta) d \eta, \\
\dot{\hat{x}}(\tau)=f(\hat{x}(\tau))+g(\hat{x}(\tau)) \gamma(\hat{x}(\tau)) .
\end{gathered}
$$

For ease of notation, we will omit the time dependencies whenever the time is clear from the context. We assume in this paper that the solution 10 is well defined and contained in $\mathcal{D}$ for all $\tau \geq 0$ so that $\rho(\hat{x}(\tau))$ is well defined. This choice of a candidate ZCBF $h$ is motivated by the fact that in (9), $h$ measures how close the state will get to the boundary of the safe set assuming $\gamma$ is used as the control input for all future time.

In Section $\amalg$ w.A saw that we could not use the Euclidean distance for a ZCBF because when a candidate ZCBF $h$ is defined as in (7), $K(x)$ could be empty for some $x \in \mathcal{D}$. In other words, although $x \in \mathcal{D}$ there was no control input available to keep the system safe. With $h$ defined in 9 , this problem is alleviated.

Theorem 2. Given a dynamical system (1) and a set $C \subset \mathcal{D}$ defined in (3) for a continuously differentiable $h$ defined in (9) with a safety function $\rho$ and locally Lipschitz evading maneuver $\gamma$, $h$ satisfies (4) for all $x \in C$. If in addition, $L_{g} h(x)$ is non-zero for all $x \in \partial C$ and $\gamma$ maps to values in the interior of $U$, then $h$ is a ZCBF on an open set $\mathcal{D}$ where $C \subset \mathcal{D}$.

Proof. We start by assuming $x \in C$ and show that $h$ satisfies (4). Because $x \in C, h(x) \geq 0$ so $\alpha(h(x)) \geq 0$. Further, note that $L_{f} h(x)+L_{g} h(x) \gamma(x)$ is the derivative along the trajectory of $\hat{x}$. In other words,

$$
L_{f} h(x)+L_{g} h(x) \gamma(x)=\lim _{a \rightarrow 0^{+}} \frac{1}{a}\left(\inf _{\tau \in[a, \infty)} \rho(\hat{x}(\tau))-\inf _{\tau \in[0, \infty)} \rho(\hat{x}(\tau))\right)
$$


Consider the term inside the parenthesis in (12), namely

$$
\inf _{\tau \in[a, \infty)} \rho(\hat{x}(\tau))-\inf _{\tau \in[0, \infty)} \rho(\hat{x}(\tau))
$$

and notice that it is the subtraction of an infimum of the same function $\rho$ evaluated on two different intervals. Further, note that the first interval is a subset of the second interval since $a$ approaches 0 from above. Thus, the term inside the parenthesis on the right hand side of $\left[12\right.$ is non-negative so $L_{f} h(x)+L_{g} h(x) \gamma(x) \geq 0$. We can then conclude that $L_{f} h(x)+L_{g} h(x) \gamma(x)+\alpha(h(x)) \geq 0$ so $\gamma(x) \in K(x)$.

Now assume that $L_{g} h(x)$ is non-zero for some $x \in \partial C$ and $\gamma$ maps to values in the interior of $U$. We will show that there is an open set $\mathcal{D}$ that is a strict superset of $C$ for which $(4)$ holds. Let $x \in \partial C$ be such that $L_{g} h(x)$ is non-zero and $B(x, \mu)$ be a ball of radius $\mu>0$ such that for all $z \in B(x, \mu) \backslash C, L_{g} h(z)$ is non-zero. Such a ball exists such that $B(x, \mu) \backslash C$ is nonempty because $L_{g} h(x)$ is continuous. Let $d(z)$ be a non-zero vector such that $d(z)+\gamma(x) \in U$ where $d(z)$ is a non-zero vector in the direction of $L_{g} h(z)$. Note that such a vector exists because $\gamma$ maps to the interior of $U$. Also note that $L_{g} h(z) d(z)>0$. Further restrict $\mu$ so that $L_{g} h(z) d(z)+\alpha(h(z)) \geq 0$ for all $z \in B(x ; \mu) \backslash C$. Note that for similar reasons discussed earlier in the proof, $L_{f} h(z)+L_{g} h(z) \gamma(z) \geq 0$. Then

$$
L_{f} h(z)+L_{g} h(z)(\gamma(z)+d(z))+\alpha(h(z)) \geq L_{g} h(x) d(z)+\alpha(h(z)) \geq 0 .
$$

Remark 1. The intuitive reason why $h$ is a ZCBF is that whenever $h(x)$ is non-negative, we have by definition a control input $\gamma$ available to keep the system safe. A geometric view is presented in Figure 1 . Note that $\gamma$ is not the output of the Quadratic Program (6). Instead, the role of $\gamma$ is to allow $h$ to be evaluated via (9).

Remark 2. Theorem 2 holds for any class $\mathcal{K}$ function $\alpha$. When $\alpha(h(x))=0$, (4) becomes $\dot{h}(x) \geq 0$. In other words, Theorem (2) can also be used to prove Lyapunov stability properties of a set by flipping the inequality.

Remark 3. We have found an error in Lemma 1 of the conference version of this paper [39] and Theorem 2 of that paper was based on that Lemma. Therefore Theorem 2 of this paper is reformulated so as not to require that Lemma.

\section{Deriving a Barrier Function for UAV Collision Avoidance}

We now consider how to calculate $h$ defined in (9) for the UAV collision avoidance problem. From Theorem 2 the only restriction on $\gamma$ and $\rho$ is that $\gamma$ is locally Lipschitz and that $h$ is continuously differentiable so there is some flexibility in choosing $\gamma$ and $\rho$. In this section we discuss two cases where we can choose $\gamma$ and $\rho$ so that $h$ can be calculated in closed form. Let the initial state for vehicle $i(i=1,2)$ be given by $\left[\begin{array}{llll}p_{i, x_{0}} & p_{i, y_{0}} & \theta_{i, 0} & p_{i, z_{0}}\end{array}\right]^{T}$. For these examples we can calculate $h$ in 9 for arbitrary initial states in closed form. Section IV] generalizes the results 


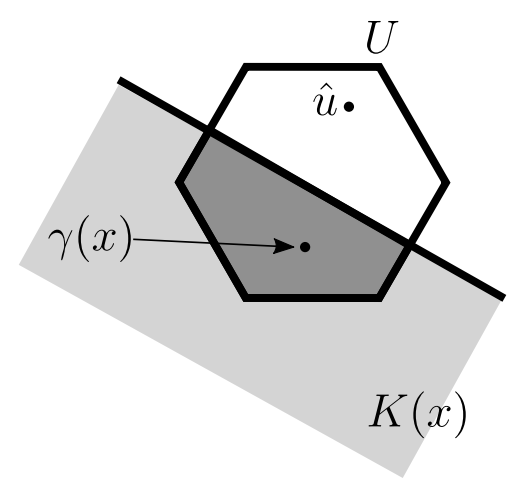

Fig. $1 h$ defined in 9 can be a barrier function because $\gamma$ or a small offset from $\gamma$ is in the intersection of $U$ and $K(x)$ for $x \in \mathcal{D}$.

from Section III.B by showing how to calculate $k(k-1) / 2$ barrier functions to ensure that the $k(k-1) / 2$ pairwise distance constraints are always satisfied. Because the examples in this section calculate $h$ in (9) using pairwise distance constraints, the calculations in these examples will also apply to the case of more than two vehicles. In other words, with the result of this section we can calculate barrier functions in closed form from arbitrary initial states and numbers of vehicles. Note that the solutions in this section solve for $h$ in $(9)$ in closed form where $\tau$ approaches infinity.

We emphasize that the specification of an evasive maneuver $\gamma$ is necessary to evaluate $h$ in (9). In other words, without a safety engineer specifying $\gamma$ there cannot be a barrier function $h$. However, $\gamma$ is never actually directly applied to the actuators. Instead, its role is to specify $h$ so that the final actuator command $u$ calculated in (6) can actually be applied to the aircraft. In this section we give two examples where for a given $\gamma, h$ can be calculated in closed form even though it is an integration over an infinite horizon. While we provide two examples of an evasive maneuver to calculate a continuously differentiable $h$ from $(9)$ in closed form, we note that it is a system specific derivation and have not identified a general method for finding a $\gamma$ for an arbitrary system that allows $h$ to be calculated in closed form. However, we emphasize that any Lipschitz continuous $\gamma$ resulting in an $h$ such that (9) can be calculated in closed form and where $h$ is continuously differentiable can be used to ensure safety via Theorem 2 While for any such $\gamma$ the safety characteristics of the system are guaranteed via Theorem 2, the performance characteristics (e.g., deviation from the nominal path) may be different for different evasive maneuvers.

Example 1. In the first case, let

$$
\gamma_{t u r n}=\left[\begin{array}{llllll}
\sigma v & \omega & 0 & v & \omega & 0
\end{array}\right]^{T}
$$

with $0<\sigma \leq 1, \omega \neq 0$. In other words, $\gamma_{\text {turn }}$ is defined by the same turn rate for both vehicles but possibly different translational velocities. See Fig. $4 \mathrm{a}$ for an example. Define $r=\frac{v}{\omega}$ to be the turn radius of the evasive maneuver when traveling at speed $v, b_{1,0}=p_{1, x_{0}}-\sigma r \sin \left(\theta_{1,0}\right), b_{2,0}=p_{2, x_{0}}-r \sin \left(\theta_{2,0}\right), c_{1,0}=p_{1, y_{0}}+\sigma r \cos \left(\theta_{1,0}\right)$, 
$c_{2,0}=p_{2, y_{0}}+r \cos \left(\theta_{2,0}\right), \Delta b_{0}=b_{1,0}-b_{2,0}, \Delta c_{0}=c_{1,0}-c_{2,0}$, and $\delta>0$. Let

$$
\rho(x)=d_{1,2}(x)-2 \delta+\delta \sin \left(\theta_{1}\right)-\delta \cos \left(\theta_{1}\right)-D_{s}^{2},
$$

where the $\delta$ terms are introduced to affect the smoothness of $h$. See the Appendix for details. Then

$$
\begin{gathered}
\left.h(x)=\inf _{\tau \in[0, \infty)}\left(\Delta b_{0}+\sigma r \sin \left(\omega \tau+\theta_{1,0}\right)-r \sin \left(\omega \tau+\theta_{2,0}\right)\right)\right)^{2}+\left(\Delta c_{0}-\sigma r \cos \left(\omega \tau+\theta_{1,0}\right)+r \cos \left(\omega \tau+\theta_{2,0}\right)\right)^{2} \\
+\left(p_{1, z_{0}}-p_{2, z_{0}}\right)^{2}-2 \delta+\delta \sin \left(\omega \tau+\theta_{1,0}\right)-\delta \cos \left(\omega \tau+\theta_{1,0}\right)-D_{s}^{2}
\end{gathered}
$$

By expanding the square terms and applying two trigonometric identities ${ }^{*}$ we get

$$
\begin{gathered}
h(x)=\inf _{\tau \in[0, \infty)} \Delta b_{0}^{2}+\Delta c_{0}^{2}+\left(1+\sigma^{2}\right) r^{2}-2 \sigma r^{2} \cos \left(\theta_{1,0}-\theta_{2,0}\right)+2 \sigma \Delta b_{0} r \sin \left(\omega \tau+\theta_{1,0}\right)-2 \Delta b_{0} r \sin \left(\omega \tau+\theta_{2,0}\right) \\
-2 \sigma \Delta c_{0} r \cos \left(\omega \tau+\theta_{1,0}\right)+2 \Delta c_{0} r \cos \left(\omega \tau+\theta_{2,0}\right) \\
+\left(p_{1, z_{0}}-p_{2, z_{0}}\right)^{2}-2 \delta+\delta \sin \left(\omega \tau+\theta_{1,0}\right)-\delta \cos \left(\omega \tau+\theta_{1,0}\right)-D_{s}^{2}
\end{gathered}
$$

Grouping constant terms and applying phasor addition yields

$$
h(x)=\inf _{\tau \in[0, \infty)} A_{1}+A_{2} \cos (\omega \tau+\Theta)-D_{s}^{2}
$$

where $A_{1}$ results from grouping constant terms, while $A_{2}$ and $\Theta$ are the amplitude and phase resulting from the phasor addition so that $A_{1}$ and $A_{2}$ are functions of $x$. By convention $A_{1}$ and $A_{2}$ are nonnegative with appropriate calculation of $\Theta$. The minimum in 16 then occurs at $\tau=(\pi-\Theta+l 2 \pi) / \omega$ for integers $l$ resulting in nonnegative $t$ so that $h(x)=A_{1}-A_{2}-D_{s}^{2}$. Note that for the case where

$$
\rho(x)=\sqrt{d_{1,2}(x)-2 \delta+\delta \sin \left(\theta_{1}\right)-\delta \cos \left(\theta_{1}\right)}-D_{s}
$$

the same reasoning yields $h(x)=\sqrt{A_{1}-A_{2}}-D_{s}$ for $\rho$ defined in 17 . To ensure that the square root is well defined, we must then require that $A_{1}-A_{2} \geq 0$ which occurs when the vehicles do not get more than $2 \delta$ from each other along the trajectory defined by (10) using $\gamma_{t u r n}$ in (13). Since $\delta$ can be chosen to be arbitrarily small, it can be chosen so that $\delta \ll D_{s}$ so the vehicles are very far outside the safe set before this condition occurs.

Example 2. For a second case, let $\rho$ be given in $(8)$ and

$$
\gamma_{\text {straight }}=\left[\begin{array}{llllll}
v_{1} & 0 & \zeta_{1} & v_{2} & 0 & \zeta_{2}
\end{array}\right]^{T},
$$

*The identities are $\sin ^{2}(\alpha)+\cos ^{2}(\alpha)=1$ and $\cos (\alpha-\beta)=\cos (\alpha) \cos (\beta)+\sin (\alpha) \sin (\beta)$. 
where $v_{1} \neq v_{2}$. In other words, $\gamma_{\text {straight }}$ uses a 0 turn rate while allowing the vehicles to have different speeds. In this case we have

$$
\begin{gathered}
h(x)=\inf _{\tau \in[0, \infty)}\left(p_{1, x_{0}}+\tau v_{1} \cos \left(\theta_{1,0}\right)-p_{2, x_{0}}-\tau v_{2} \cos \left(\theta_{2,0}\right)\right)^{2}+\left(p_{1, y_{0}}+\tau v_{1} \sin \left(\theta_{1,0}\right)-p_{2, y_{0}}-\tau v_{2} \sin \left(\theta_{2,0}\right)\right)^{2} \\
+\left(p_{1, z_{0}}+\tau \zeta_{1}-p_{2, z_{0}}-\tau \zeta_{2}\right)^{2}-D_{s}^{2}
\end{gathered}
$$

which is quadratic in $t$ so the minimum can be calculated in closed form. See the Appendix for an analysis of the differentiability of $h$ in this case.

The evasive maneuvers in 13 and $(18)$ (when $\zeta_{1}=\zeta_{2}=0$ in $(18)$ both encode trajectories where the vehicles maintain the same altitude for all times and therefore appear to not be exploiting an important evasive capability of the aircraft, namely the ability to change altitudes. However, this is not actually the case. Although $\gamma_{\text {turn }}$ and $\gamma_{\text {straight }}$ (for $\left.\zeta_{1}=\zeta_{2}=0\right)$ are purely planar maneuvers, they nevertheless can induce behaviors that exploit altitude changes. To see this, note that for $h$ in (16) and (19),

$$
\frac{\partial h(x)}{\partial p_{1, z_{0}}}=2\left(p_{1, z_{0}}-p_{2, z_{0}}\right)
$$

which is not equal to zero for $p_{1, z_{0}} \neq p_{2, z_{0}}$. A similar calculation also holds for $\frac{\partial h(x)}{\partial p_{2, z_{0}}}$. In other words, $h$ changes as a function of initial altitude. Specifically, this means that the QP can exploit $\zeta_{1}$ and $\zeta_{2}$ because the fourth and eighth elements of $L_{g} h(x(t))$ are non-zero when $p_{1, z_{0}} \neq p_{2, z_{0}}$, i.e., the QP in 6 can exploit the altitude control input even though $\gamma_{\text {turn }}$ and $\gamma_{\text {straight }}$ do not necessarily include an altitude changing term in the evasive maneuver.

\section{Simulation of Two Vehicles}

We demonstrate the theoretical development of this section in simulation using SCRIMMAGE [40]. SCRIMMAGE is a multi-agent simulator designed to scale to high numbers of vehicles and includes a plugin-interface that makes it easy to experiment with different motion models and controllers without having to change code. This makes it simple to swap out nominal controllers and vary the fidelity of fixed-wing UAVs from the unicycle dynamics in (2) used in this section up to a 6-DOF model.

For the simulation, let $k$ vehicles be positioned in a circle of radius 200 around the origin, where $k=2$ in this

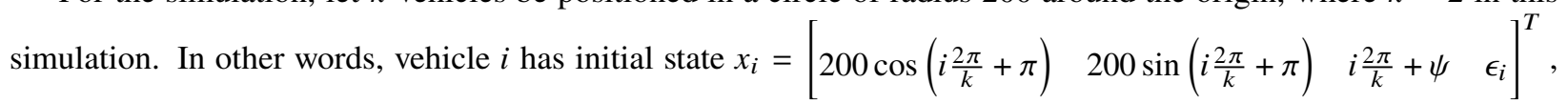
where $\psi$ is an additional offset so that vehicles are not necessarily starting with orientation pointing at the origin. The goal position for vehicle $i$ is on the other side of the origin: $x_{i, g}=\left[200 \cos \left(i \frac{2 \pi}{k}\right) \quad 200 \sin \left(i \frac{2 \pi}{k} \pi\right)\right]^{T}$.

This setup is selected so that the vehicles are on a collision course. The nominal controller is that described in [41] with constant $\lambda=1$. Additionally, we let $v_{\min }=15$ meters $/$ second, $v_{\max }=25$ meters $/$ second, $\zeta_{\max }=3.9$ meters/second, $\omega_{\max }=13$ degrees/second, $D_{s}=5$ meters, and $\delta=0.01$ meters ${ }^{2}$. The choice of $\zeta_{\max }$ results from 
assuming a maximum pitch of 15 degrees while traveling at $v_{\min }$. $\omega_{\max }$ is chosen to be consistent with a constant rate turn [42] with a 30 degree bank with a speed of $v_{\max }$. We note that while the experiments do not consider dynamics or sensor noise, the robustness of barrier functions to noise was previously discussed in [29]. Each vehicle evaluates [6] at each timestep where we use OSQP [43] to evaluate the QP. We investigate the performance of the vehicles when $h$ defined in 99 is constructed from $\gamma_{\text {turn }}$ in 13 and $\gamma_{\text {straight }}$ 18, , respectively, where $\gamma_{\text {turn }}=\left[\begin{array}{llllll}v & \omega & 0 & v & \omega & 0\end{array}\right]^{T}$, $\gamma_{\text {straight }}=\left[\begin{array}{llllll}v & 0 & 0 & v & 0 & 0\end{array}\right]^{T}$, and $v=0.9 v_{\min }+0.1 v_{\max }$ and $\omega=0.9 \omega_{\max }$. For the scenario with $\gamma_{\text {turn }}$, we let $\psi=0$ so that the vehicles start with orientation pointing at the origin. For the scenario with $\gamma_{\text {straight }}$, we let $\psi=2^{\circ}$ because if the vehicles pointed at the origin they would not start in the safe set. Additionally, for the $\gamma_{\text {turn }}$ case we use $\rho$ in 177. Similarly, for the $\gamma_{\text {straight }}$ case we use $\rho(x)=\sqrt{d_{1,2}(x)}-D_{s}$. Details of the distance between the vehicles and control signals are shown in Figure 2 Note that the resulting trajectory can be different depending on which $\gamma$ is used as shown in Figure 2d Nevertheless, in both cases the vehicles are able to maintain safe distances from each other and satisfy actuator constraints throughout the simulation regardless of which $\gamma$ is used to construct a $h$.

In the second experiment, we examine the effect of altitude control on the evasive behavior of the aircraft. Because 20 predicts that $\frac{\partial h(x)}{\partial p_{i, z_{0}}} \neq 0$ (for $i=1,2$ ) only when the vehicles are not at the same altitude, we start the vehicles at an altitude of -1 and 1, respectively. This offset is small enough to ensure that the nominal path of the vehicles still involves a collision. As was done in the previous experiment, we set $\psi=0^{\circ}$ and $\psi=2^{\circ}$ degrees when using $\gamma_{\text {turn }}$ and $\gamma_{\text {straight }}$, respectively. In Figure 2 we show the output of $\zeta_{1}$, where overriding behavior peaks around 8.2 seconds. Notice that the actuator output is within the limits of $\pm \zeta_{\max }$. Further, the vehicles maintain safe distances at all times. This occurs even though the evading maneuver does not explicitly encode altitude changes.

\section{COMPOSITION OF MULTIPLE SAFETY CONSTRAINTS}

\section{A. Motivating Example}

Although the constructive method introduced in (9) can produce a barrier function in the presence of actuator constraints that ensures two vehicles do not collide, the formulation does not extend immediately to collision avoidance for systems with more than two vehicles. To see this, we present a specific example where three UAVs with a collision avoidance safety objective cannot use the results from Section III.B to ensure safety. A plot of this scenario is shown in Figure 4. We index the vehicles by $i=1,2,3$. To ensure collision-free trajectories, and considering the safety function defined in (14), three pairwise constraints must be nonnegative at all times:

$$
\begin{aligned}
& \rho^{1}(x)=d_{1,2}(x)-2 \delta+\delta \sin \left(\theta_{1}\right)-\delta \cos \left(\theta_{1}\right)-D_{s}^{2}, \\
& \rho^{2}(x)=d_{1,3}(x)-2 \delta+\delta \sin \left(\theta_{1}\right)-\delta \cos \left(\theta_{1}\right)-D_{s}^{2}, \\
& \rho^{3}(x)=d_{2,3}(x)-2 \delta+\delta \sin \left(\theta_{2}\right)-\delta \cos \left(\theta_{2}\right)-D_{s}^{2} .
\end{aligned}
$$




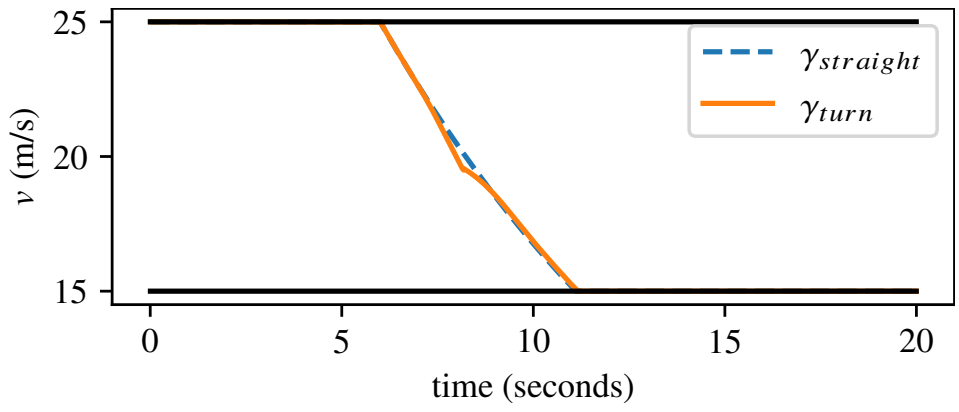

(a)

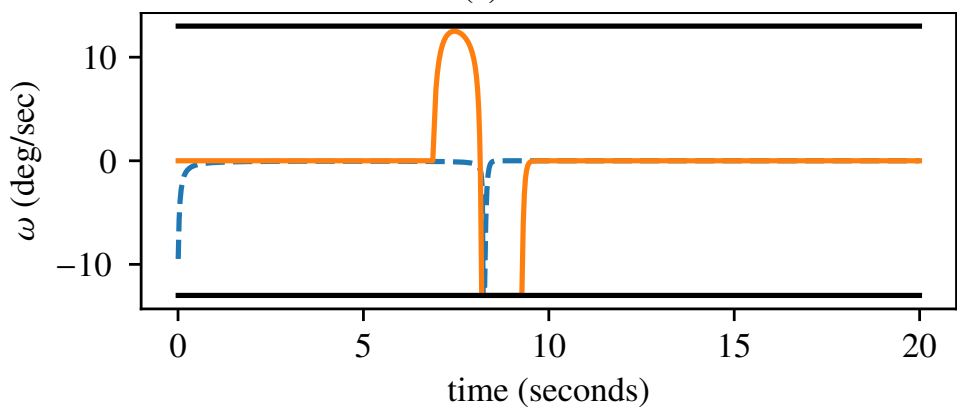

(b)

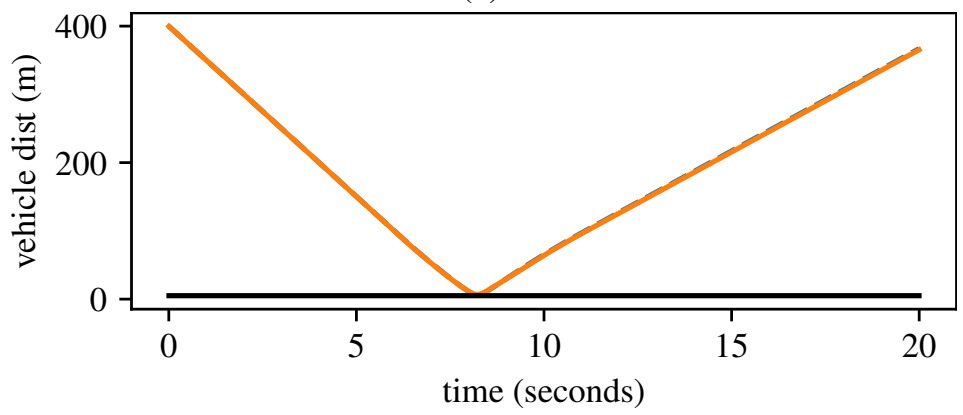

(c)

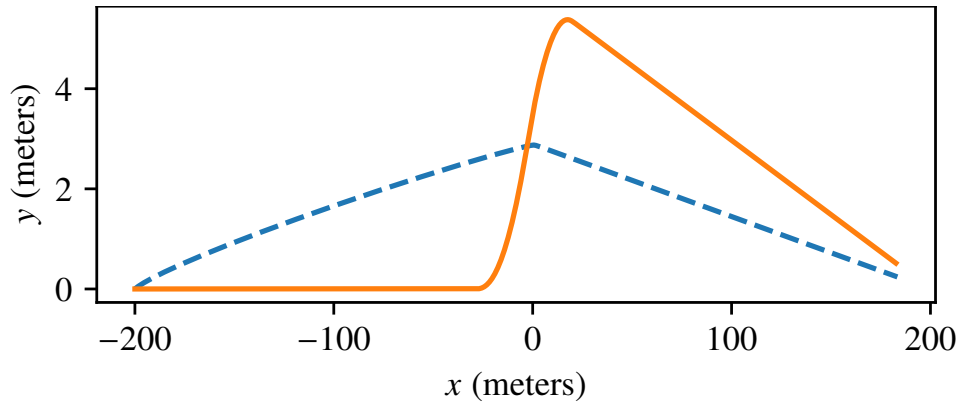

(d)

Fig. 22 vehicle scenario statistics. (a) vehicle 1 velocity, (b) vehicle 1 turn rate, (c) intervehicle distance, (d) vehicle 1 path. Adapted with permission from [39] C2018 IEEE. 


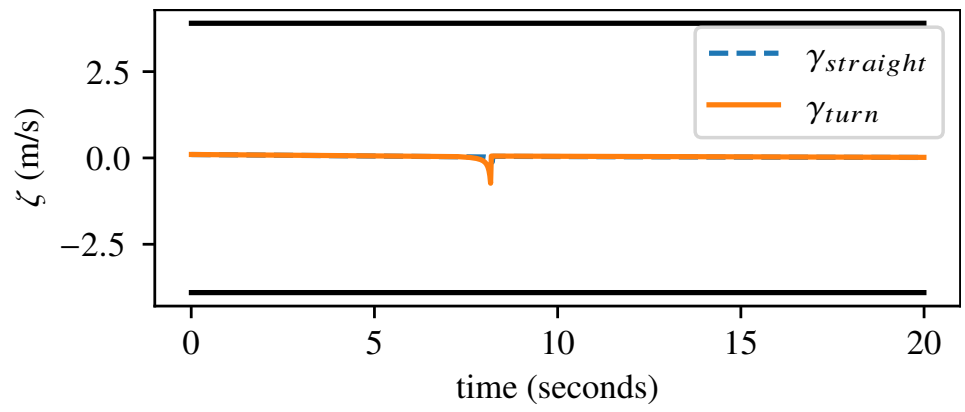

(a)

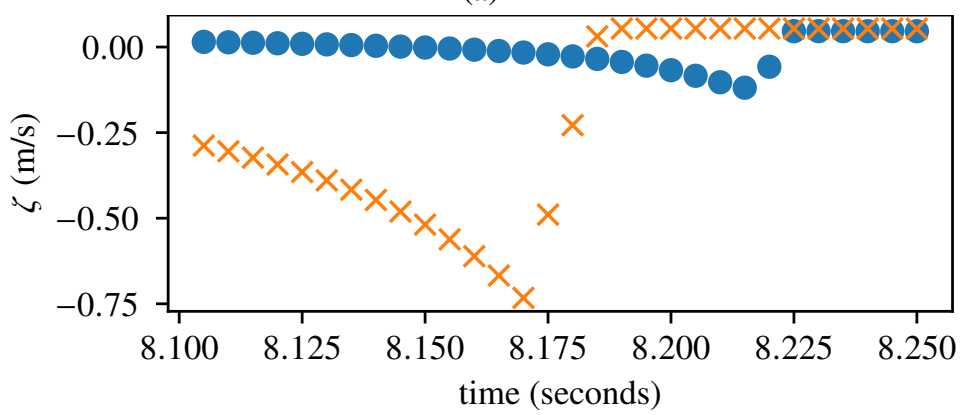

(b)

Fig. 3 (a) $\zeta_{1}$ output for barrier functions based on $\gamma_{t u r n}$ and $\gamma_{\text {straight }}$ where an override is apparent around 8.2 seconds. (b) A zoomed in plot of the override.

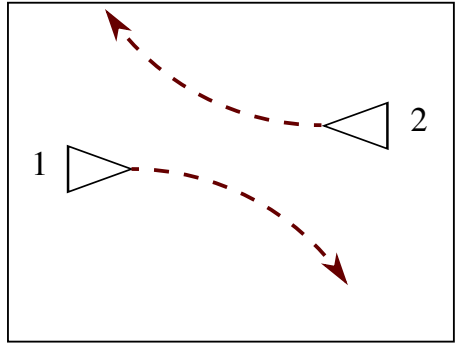

(a)

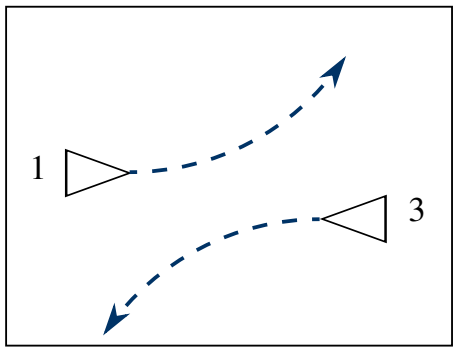

(b)

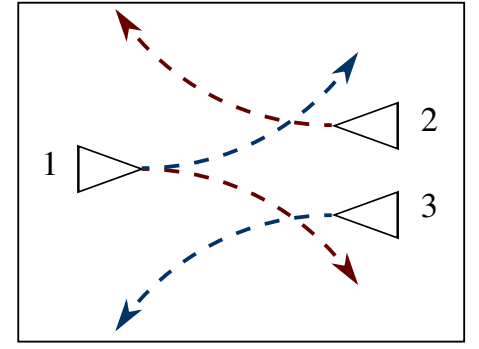

(c)

Fig. 4 A geometric view of the example given in Section IV.A.

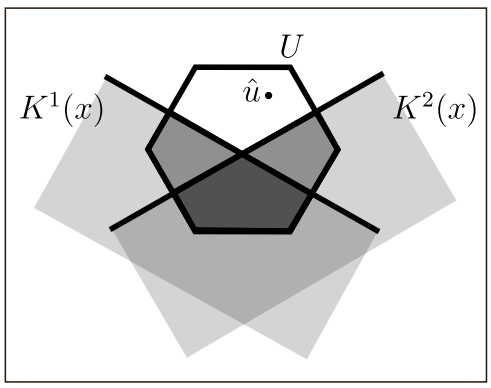

(a)

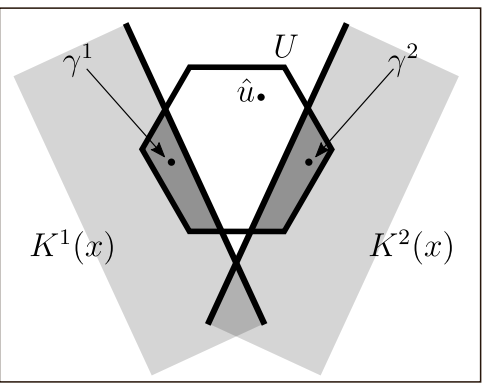

(b)

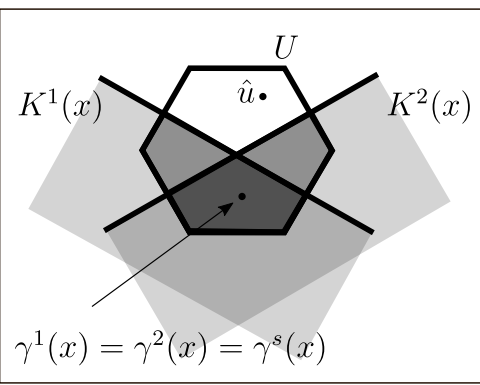

(c)

Fig. 5 The shared evading maneuver ensures that the same safe control input satisfies each safety constraint. 
We now apply the results of Section III to these constraints and for simplicity, let $\delta$ be approximately 0 . For each constraint, define an arbitrarily chosen nominal evading maneuver

$$
\begin{aligned}
& \gamma^{1}(x)=\left[\begin{array}{llllllllll}
1 & -1 & 0 & 1 & -1 & 0 & 1 & -1 & 0
\end{array}\right]^{T} \\
& \gamma^{2}(x)=\gamma^{3}(x)=\left[\begin{array}{lllllllll}
1 & 1 & 0 & 1 & 1 & 0 & 1 & 1 & 0
\end{array}\right]^{T} .
\end{aligned}
$$

In other words, $\gamma^{1}$ encodes an evasive maneuver where all the vehicles turn right while $\gamma^{2}$ and $\gamma^{3}$ encode a maneuver where all the vehicles turn left. We note that $h^{j}(j=1, \ldots, 3)$ defined in 9 and constructed from $\rho^{j}$ and $\gamma^{j}$ are ZCBFs. In this example we let $v_{\min }=1, v_{\max }=2, \omega_{\max }=1$, and $D_{s}=0.5$ so that the vehicles follow a circular trajectory with radius $r=1$ when applying $v_{\min }$ and $\omega_{\max }$. Assume now that the vehicles have the following initial states

$$
\begin{aligned}
& x_{1}=\left[\begin{array}{llll}
0 & 0 & 0 & 0
\end{array}\right]^{T}, \\
& x_{2}=\left[\begin{array}{llll}
\left(2 r+D_{s}\right) \sin \psi & \left(2 r+D_{s}\right) \cos \psi-2 r & \pi & 0
\end{array}\right]^{T}, \\
& x_{3}=\left[\begin{array}{llll}
\left(2 r+D_{s}\right) \sin \psi & 2 r-\left(2 r+D_{s}\right) \cos \psi & \pi & 0
\end{array}\right]^{T},
\end{aligned}
$$

where $\psi=\arccos \left(\frac{D_{s} / 2+2 r}{2 r+D_{s}}\right)$. Then $h^{1}(x)=h^{2}(x)=h^{3}(x)=0$ and the barrier constraints in 4 for $h^{1}(x)$ and $h^{2}(x)$ become

$$
\begin{aligned}
& -0.4\left(v_{1}+\omega_{1}+v_{2}+\omega_{2}\right) \geq 0 \\
& 0.4\left(-v_{1}+\omega_{1}-v_{3}+\omega_{3}\right) \geq 0 .
\end{aligned}
$$

Although $h^{1}$ and $h^{2}$ are ZCBFs, these two constraints cannot be simultaneously satisfied for $v_{i} \in\left[v_{\min }, v_{\max }\right]$ and $\left|\omega_{i}\right| \leq \omega_{\max }$. In particular, after substituting the minimum velocity $v_{1}=v_{2}=1$, the first equation dictates that $\omega_{1}+\omega_{2} \leq-2$ (i.e., vehicles 1 and 2 must turn right). Similarly, the second equation dictates that vehicle 1 and 3 must turn left. The problem with this scenario is that vehicle 1 cannot simultaneously execute both nominal evading maneuvers (i.e., turn both left and right at the same time). To solve this problem, we will make sure that the evasive maneuver applied by a vehicle is the same for every barrier function. A geometric view of the general problem and its solution are shown in Figure 5 .

\section{B. Sufficient Conditions for Satisfying Multiple Safety Constraints}

In order to solve the issues arising when vehicles have to simultaneously respect multiple constraints, we now extend the use of the constructive technique introduced in [9]. In this section we extend the reasoning of [23] to the case of $q$ 
constraints. Consider a nonlinear autonomous system

$$
\dot{x}=f(x)
$$

where $f$ is locally Lipschitz. Then we have a similar definition to Definition 1 for autonomous systems.

Definition 2. [23] Given a set $C \subset \mathbb{R}^{n}$ defined in (3) for a continuously differentiable function $h: \mathbb{R}^{n} \rightarrow \mathbb{R}$, the function $h$ is called a zeroing barrier function $(Z B F)$ defined on an open set $\mathcal{D}$ with $C \subset \mathcal{D} \subset \mathbb{R}^{n}$, if there exists a Lipschitz continuous extended class $\mathcal{K}$ function $\alpha$ such that

$$
L_{f} h(x) \geq-\alpha(h(x)), \forall x \in \mathcal{D} \text {. }
$$

When there are $q$ constraints, we consider the case of $q$ barrier functions where each barrier function is denoted $h^{j}$ on $\mathcal{D}^{j}$ with associated safe set $C^{j}$ and admissible control space $K^{j}(x)$ for $x \in \mathcal{D}^{j}$ for $j \in\{1, \ldots, q\}$. We are interested in the conditions under which all safety constraints can be satisfied for all future times. In other words, under the assumption that $x(0) \in C^{j}$ we want to show that $x(t) \in C^{j}$ for all $t \geq 0$. Hence, we are interested in the forward invariance of the intersection of all the safe sets, which motivates the following definitions

$$
\begin{aligned}
C_{\cap} & =C^{1} \cap C^{2} \cap \cdots \cap C^{q}, \\
K_{\cap}(x) & =\left\{u \in U: u \in K^{1}(x) \cap K^{2}(x) \cap \cdots \cap K^{q}(x)\right\} .
\end{aligned}
$$

where $\mathcal{D}_{\cap}$ is an open superset of $\mathcal{C}_{\cap}$ and $x \in \mathcal{D}_{\cap}$. We can now present a multiple constraint analogue of Theorem 1 by following the same reasoning as [23].

Proposition 1. Given a dynamical system (23) and a set $C_{\cap}$ defined by (25) for continuously differentiable functions $h^{j}: R^{n} \rightarrow R$ where $h^{j}$ is a ZBF on $D^{j}$ with $C^{j} \subset D^{j} \subset R$ and $\frac{\partial h^{j}(x)}{\partial x} \neq 0$ for any $x \in \partial C_{\cap}$ where $h^{j}(x)=0$, then $C_{n}$ is forward invariant.

Proof. The proof is the same as that for Proposition 1 of [23], namely $\dot{h}^{j}(x)=-\alpha(x) \geq 0$ for any $j$ such that $h^{j}(x)=0$ so the result follows by Nagumo's Theorem [44]. We add the assumption that $\frac{\partial h^{j}(x)}{\partial x}$ is non-zero for all $x \in \partial \mathcal{C}_{\cap}$ such that $h^{j}(x)=0$ to ensure that the tangent cone in Nagumo's Theorem is non-empty.

Then for autonomous systems with dynamics (1), we have the following corollary of Theorem 1 .

Corollary 1. Given a dynamical system (1) and a set $\mathcal{C}_{\cap}$ defined by (25) for continuously differentiable functions 
$h^{j}: R^{n} \rightarrow R$ where $h^{j}$ is a ZCBF on $D^{j}$ and $\frac{\partial h^{j}(x)}{\partial x} \neq 0$ for any $x \in \partial \mathcal{C}_{\cap}$ where $h^{j}(x)=0$, then any Lipschitz continuous controller $u: \mathcal{D}_{\cap} \rightarrow U$ such that $u(x) \in K_{\cap}(x)$ will render the set $C_{\cap}$ forward invariant.

\section{The Shared Nominal Evading Maneuver Assumption}

Suppose there are $q$ constraints $\rho^{j}: \mathcal{D}^{j} \rightarrow \mathbb{R}(j=1, \ldots, q)$ that must be greater than or equal to 0 at all times. For the $k$ agents with pairwise constraints $q=k(k-1) / 2$. We assume that for each constraint $j=1, \ldots, q$, a locally Lipschitz nominal evading maneuver $\gamma^{j}$ has been selected using the framework in 97. An example for fixed-wing UAVs with collision avoidance safety constraints is given in 13 . Given $q$ safety functions $\rho^{j}$ and evading maneuvers $\gamma^{j}$ for $j \in\{1, \ldots, q\}$, we construct $q$ output functions $h^{j}$ defined on $\mathcal{D}^{j}$ similarly to 9$\}$ where

$$
\begin{aligned}
h^{j}(x ; \rho, \gamma) & =\inf _{\tau \in[0, \infty)} \rho^{j}\left(\hat{x}^{j}(\tau)\right), \\
\hat{x}^{j}(\tau) & =x+\int_{0}^{\tau} \dot{\hat{x}}^{j}(\eta) d \eta, \\
\dot{x}^{j}(\tau) & =f\left(\hat{x}^{j}(\tau)\right)+g\left(\hat{x}^{j}(\tau)\right) \gamma^{j}\left(\hat{x}^{j}(\tau)\right) .
\end{aligned}
$$

Section IV.A showed an example where $K_{n}$ could be empty for some $x \in C_{n}$. As a result, the assumptions of Corollary 1 could not be satisfied. In order to address the issue discussed in Section IV.A. we introduce an additional constraint on $\gamma^{j}(j=1, \ldots, q)$ that all $h^{j}$ are constructed from the same nominal evading maneuver.

Assumption 1. Given a dynamical system 11 and $q$ output functions $h^{j}$ defined in 27) for given safety functions $\rho^{j}$ and evading maneuvers $\gamma^{j}$ for $j \in\{1, \ldots, q\}$, the shared evading maneuver assumption holds if $\gamma^{1}(x)=\cdots=\gamma^{q}(x)$ for all $x \in \mathcal{D}_{\cap}$. The shared evading maneuver is denoted $\gamma^{s}$ so that

$$
\gamma^{s}(x)=\gamma^{1}(x)=\cdots=\gamma^{q}(x)
$$

for all $x \in \mathcal{D}_{\cap}$.

Remark 4. This assumption requires that each $h^{j}(j=1, \ldots, q)$ be constructed from the same nominal evading maneuver. Note, however, that this does not imply that each $h^{j}$ must be constructed from the same safety function.

The example in Section IV.A does not satisfy Assumption 1 because $\gamma^{1}(x)$ and $\gamma^{2}(x)$ defined in 21, are not the same. To enforce that the shared evasive maneuver assumption holds, one option is to change $\gamma^{1}$ so that

$$
\gamma^{1}(x)=\left[\begin{array}{lllllllll}
1 & 1 & 0 & 1 & 1 & 0 & 1 & 1 & 0
\end{array}\right]^{T}
$$

In other words, using $\gamma^{1}$ defined in 31 and $\gamma^{2}$ and $\gamma^{3}$ in 20b implies an evasive maneuver where all vehicles turn left 
for each constraint. Another example where the shared nominal evading maneuver assumption holds is as follows:

$$
\begin{gathered}
\gamma^{s}(x)=\gamma^{1}(x)=\gamma^{2}(x)=\gamma^{3}(x)= \\
{\left[\begin{array}{lllllllll}
1 & 1 & 0 & 1.5 & 0 & 0 & 2 & -1 & 0
\end{array}\right]^{T} .}
\end{gathered}
$$

In this case, $\gamma^{s}(x)$ encodes an evasive maneuver where vehicle 1 turns left with a linear velocity of 1 , vehicle 2 stays straight with a linear velocity of 1.5 , and vehicle 3 turns right with a linear velocity of 2 . These three nominal evading maneuvers satisfy the shared evasive maneuver assumption because for all $x \in \mathcal{D}_{\cap}, \gamma^{1}(x)=\gamma^{2}(x)=\gamma^{3}(x)$.

To see the purpose of Assumption 1. we first examine the case of a single constraint. In particular, let $h$ be defined in (9) and consider the role of $\gamma$ in establishing that $h$ is a ZCBF. From Definition 1 , for $h$ to be used for a barrier function, $K(x)$ must be nonempty for all $x \in \mathcal{D}$. With $h$ defined as in 9 , this property is satisfied by $\gamma(x)$ or a perturbation of $\gamma(x)$ for all $x \in \mathcal{D}$ (see Theorem 2). The analogue condition for multiple constraints is that $K_{\cap}(x)$ is non-empty for all $x \in \mathcal{D}_{\cap}$. If each $h^{j}$ defined in 9$]$ is a ZCBF and is constructed from $\gamma^{j}$ then by similar reasoning to Theorem $2, \gamma^{j}(x)$ or a perturbation of $\gamma(x)$ is in $K^{j}(x)$ for all $x \in \mathcal{D}_{\cap}$. This allows us to state a multiple constraint analogue to Theorem 2 In the following, we denote the inner product as $\left\langle L_{g} h^{j_{1}}(x), L_{g} h^{j_{2}}(x)\right\rangle$ for $j_{1}, j_{2} \in\{1, \ldots, q\}$.

Theorem 3. Given a dynamical system (1) and a set $\mathcal{C}_{\cap} \subset \mathcal{D}_{\cap}$ defined in (25) for $q$ continuously differentiable functions $h^{j}$ defined in (27) with safety functions $\rho^{j}$ and evading maneuvers $\gamma^{j}$ where $k \in\{1, \ldots, q\}$, if $h^{j}$ is a ZCBF for $k \in\{1, \ldots, q\}$ and Assumption 1 holds then $K_{\cap}(x)$ is non-empty for all $x \in C_{\cap}$. If in addition, $\gamma^{s}$ defined in 30 maps to the interior of $U$ and for all $x \in \partial C_{\cap},\left\langle L_{g} h^{j_{1}}(x), L_{g} h^{j_{2}}(x)\right\rangle>0$ for $j_{1} \neq j_{2}$ and $j_{1}, j_{2} \in\{1, \ldots, q\}$, then there is an open set that is a superset of $C_{\cap}$ for which $K_{\cap}(x)$ is non-empty for all $x$ in the open set.

Proof. To prove the first statement, note that it was shown in the proof of Theorem 2 that $\gamma^{s}$ is in $K^{j}(x)$ for $j=1, \ldots, q$ and $x \in C_{\cap}$. To prove the second statement, note that we can use the same method as was used in the proof of Theorem 2 to find a vector $d(z)$ such that $h^{j}(z)$ satisfies 4 for all $z \in B(x, \mu)$ given $x \in \partial C_{\cap}$. In particular, because $\left\langle L_{g} h^{j_{1}}(x), L_{g} h^{j_{2}}(x)\right\rangle>0, L_{g} h^{j}(x) \neq 0$ for $j=1, \ldots, q$ there exists a vector $d_{\text {all }}(x)$ such that $\left\langle d_{\text {all }}(x), L_{g} h^{j}(x)\right\rangle>0$. We choose $d_{\text {all }}(x)$ with sufficiently small norm. Using the notation of the proof of Theorem 2 , for sufficiently small $\mu$, the projection of $d_{\text {all }}(x)$ onto $L_{g} h(z)$ will be in the direction of $L_{g} h(z)$ for $z \in B(x, \mu)$ because $L_{g} h(x)$ is continuous.

Remark 5. A geometric view of the problem introduced in Section IV.A and its resolution via the shared evading maneuver assumption is shown in Figure 5

Similar to the QP in 46 , we write a QP with $q$ constraints and let $\hat{u}=\left[\begin{array}{llll}\hat{u}_{1}^{T} & \hat{u}_{2}^{T} & \cdots & \hat{u}_{k}^{T}\end{array}\right]^{T}$ where $\hat{u}_{i}$ is the nominal input of vehicle $i$ for $i=1, \ldots, k$. To emphasize that all $h^{j}$ are constructed from $\gamma^{s}$, we write $h^{j, s}$ for each $j=1, \ldots, q$ 
as follows:

$$
\begin{array}{ll} 
& u^{*}=\min _{u \in \mathbb{R}^{m}} \frac{1}{2}\|u-\hat{u}\|^{2} \\
\text { s.t. } & A u \geq b . \\
& \quad L_{f} h^{j, s}(x)+L_{g} h^{j, s}(x) u+\alpha\left(h^{j, s}(x)\right) \geq 0 \quad j \in\{1, \ldots, q\} .
\end{array}
$$

\section{CONTROL CALCULATION WITH LIMITED COMMUNICATION}

The QP in 32 is a centralized calculation. In particular, it requires that each vehicle's nominal control input $\hat{u}_{i}$ be communicated. Frequently communicating this signal when there are many vehicles may reduce throughput for other important messages or introduce communication delays because a network can only support a limited number of bits per second through a network. Thus, we show how to ensure safety constraints can be satisfied by reformulating the QP so that the vehicles can calculate a safe control signal without requiring each other's nominal control input. However, we continue to assume that each vehicle can sense the state of every other vehicle.

We start by considering the two vehicle case and then generalize to the $k$ vehicle case. Let $\gamma^{s}=\left[\begin{array}{l}\gamma_{1}^{s^{T}} \\ \gamma_{2}^{s^{T}}\end{array}\right]^{T}$ be the shared evading maneuver where $\gamma_{1}^{s^{T}}$ is the part of $\gamma^{s}$ that is applied to vehicle 1 and therefore has the same size as $u_{1}$. Define $\gamma_{2}^{s}$ similarly for vehicle 2. Similarly decompose $b$ in 6 c $b$ as $b=\left[\begin{array}{ll}b_{1}^{T} & b_{2}^{T}\end{array}\right]^{T}$ and $L_{g} h^{j, s}(x)$ as $L_{g} h^{j, s}(x)=\left[\left[L_{g} h^{j, s}(x)\right]_{1}^{T} \quad\left[L_{g} h^{j, s}(x)\right]_{2}^{T}\right]^{T}$. Further, let $A$ in $\quad 6 c$ be block diagonal with block entries $A_{1}$ and $A_{2}$ so that $A_{i} u_{u} \geq b_{i}$ represents the actuator constraint for vehicle $i$ for $i=1,2$.

We want to find a way of calculating $u_{1}$ and $u_{2}$ such that $u=\left[\begin{array}{ll}u_{1}^{T} & u_{2}^{T}\end{array}\right]^{T}$ satisfies $A u \geq b$ and $u \in K^{j}(x)$ for all $x \in \mathcal{D}$ where the calculation for $u_{1}$ does not require knowledge of $\hat{u}_{2}$ or the final value for $u_{2}$. Similarly, we want to calculate $u_{2}$ without knowledge of $\hat{u}_{1}$ or $u_{1}$. This is a trivial requirement for actuator constraints since $A_{i} u_{i} \geq b_{i}$ for $i=1,2$ if and only if $A u \geq b$. However, the constraint that $u \in K_{\cap}(x)$ involves both $u_{1}$ and $u_{2}$ so we reformulate it as follows:

$$
\begin{aligned}
0 & \leq L_{f} h^{j, s}(x)+L_{g} h^{j, s}(x) u+\alpha\left(h^{j, s}(x)\right) \\
& =\kappa_{1}\left(x, u_{1}\right)+\kappa_{2}\left(x, u_{2}\right)
\end{aligned}
$$

where

$\kappa_{1}\left(x, u_{1}\right)=L_{f} h^{j, s}(x)+\left[L_{g} h^{j, s}(x)\right]_{1} u_{1}+\alpha\left(h^{j, s}(x)\right)+\left[L_{g} h^{j, s}(x)\right]_{2} \gamma_{2}^{s}-\frac{1}{2}\left(L_{f} h^{j, s}(x)+L_{g} h^{j, s}(x) \gamma^{s}+\alpha\left(h^{j, s}(x)\right)\right)$ 
and

$\kappa_{2}\left(x, u_{2}\right)=L_{f} h^{j, s}(x)+\left[L_{g} h^{j, s}(x)\right]_{2} u_{2}+\alpha\left(h^{j, s}(x)\right)+\left[L_{g} h^{j, s}(x)\right]_{1} \gamma_{1}^{s}-\frac{1}{2}\left(L_{f} h^{j, s}(x)+L_{g} h^{j, s}(x) \gamma^{s}+\alpha\left(h^{j, s}(x)\right)\right)$

Notice that $\kappa_{1}$ is not a function of $u_{2}$ and $\kappa_{2}$ is not a function of $u_{1}$. In other words, if we can select $u_{1}$ and $u_{2}$ such that $\kappa_{1}\left(x, u_{1}\right) \geq 0$ and $\kappa_{2}\left(x, u_{2}\right) \geq 0$ then $u=\left[\begin{array}{ll}u_{1}^{T} & u_{2}^{T}\end{array}\right]^{T} \in K_{\cap}(x) \forall x \in C$. For $x \in C_{\cap}$, this can be done by letting $u_{1}=\gamma_{1}^{s}(x)$ and $u_{2}=\gamma_{2}^{s}(x)$ and noting that this implies

$$
\kappa_{1}\left(x, \gamma_{1}^{s}\right)+\kappa_{2}\left(x, \gamma_{2}^{s}\right)=L_{f} h^{j, s}(x)+L_{g} h^{j, s}(x) \gamma^{s}+\alpha\left(h^{j, s}(x)\right) \geq 0
$$

For $x \notin C_{\cap}$, a pertubation of $\gamma_{1}^{s}(x)$ and $\gamma_{2}^{s}(x)$ using a similar method as shown in the proof of Theorem 3 suffices. In other words, we can find $u$ without vehicle 1 needing to know $\hat{u}_{2}$ or $u_{2}$ and similarly for vehicle 2 . Each vehicle $i$ $(i=1,2)$ could then calculate the following $\mathrm{QP}$ :

$$
\begin{array}{cc}
u^{*}= & \min _{u \in \mathbb{R}^{m_{i}}} \frac{1}{2}\left\|u-\hat{u}_{i}\right\|^{2} \\
\text { s.t. } & A_{i} u_{i} \geq b_{i} \\
& \kappa_{i}\left(x, u_{i}\right) \geq 0 .
\end{array}
$$

Note that $\kappa_{i}\left(x, u_{i}\right)$ is linear in $u_{i}$.

We now generalize the above discussion to $k$ vehicles. Let $\gamma^{s}=\left[\begin{array}{lll}\gamma_{1}^{s^{T}} & \cdots & \gamma_{k}^{s^{T}}\end{array}\right]^{T}$, where $\gamma_{i}^{s}$ maps to vectors of the same size as $u_{i}$ for $i=1, \ldots, k$ with similar decomposition for $b=\left[\begin{array}{lll}b_{1}^{T} & \cdots & b_{k}^{T}\end{array}\right]^{T}$ and $L_{g} h^{j, s}(x)=$ $\left[\begin{array}{lll}{\left[L_{g} h^{j, s}(x)\right]_{1}^{T}} & \cdots & {\left[L_{g} h^{j, s}(x)\right]_{k}^{T}}\end{array}\right]^{T}$. Further, assume $A$ in $6 \mathrm{cc}$ is block diagonal with block entries $A_{i}$ for $i=1, \ldots, k$ where $A_{i}$ is a $m_{i} \times m_{i}$ matrix. This assumption means that actuator constraints are not coupled between vehicles. For constraint $j$ for $j=1, \ldots, q$, let

$$
\mathcal{V}^{j}=\left\{i \in\{1, \ldots, k\}: \exists x \in \mathcal{D} \text { s.t. }\left[L_{g} h^{j, s}(x)\right]_{i} \neq 0_{m_{i}}\right\}
$$

where $0_{m_{i}}$ is the zero vector in $\mathbb{R}^{m_{i}} \cdot \mathcal{V}^{j}$ represents the set of vehicles whose control input affects the time derivative of $h^{j}$ for some $x \in \mathcal{D}$. We let $\left|\mathcal{V}^{j}\right|$ denote the cardinality of $\mathcal{V}^{j}$, and note that for the case of pairwise collision avoidance, $\left|\mathcal{V}^{j}\right|=2$ for all $j=1, \ldots, q$. In the example with three vehicles in Section IV $\mathcal{V}_{1}=\{1,2\}, \mathcal{V}_{2}=\{1,3\}, \mathcal{V}_{3}=\{2,3\}$ Finally, we denote $u_{\backslash i}=\left[\begin{array}{llllll}u_{1}^{T} & \cdots & u_{i-1}^{T} & u_{i+1}^{T} & \cdots & u_{k}^{T}\end{array}\right]^{T}$, with similar definitions for $\gamma_{\backslash i}^{s}, \hat{u}_{\backslash i}$, and $\left[L_{g} h^{j, s}(x)\right]_{\backslash i}$.

With the above definitions, we can now state a limited communication analogue for the admissible control space in 5 . The limited communication admissible control space for constraint $j(j=1, \ldots, q)$ and vehicle $i\left(i \in \mathcal{V}^{j}\right)$ is 
defined as

$$
\begin{aligned}
\mathcal{K}_{i}^{j}(x)=\left\{u_{i} \in U_{i}: 0 \leq\right. & L_{f} h^{j, s}(x)+\left[L_{g} h^{j, s}(x)\right]_{i} u_{i}+\alpha\left(h^{j, s}(x)\right)+\left[L_{g} h^{j, s}(x)\right]_{\backslash i} \gamma_{\backslash i}^{s}(x) \\
& \left.-\frac{\left|\mathcal{V}^{j}\right|-1}{\left|\mathcal{V}^{j}\right|}\left(L_{f} h^{j, s}(x)+L_{g} h^{j, s}(x) \gamma^{s}(x)+\alpha\left(h^{j, s}(x)\right)\right)\right\} .
\end{aligned}
$$

Let $\mathcal{S}_{i}=\left\{j \in\{1, \ldots, q\}: i \in \mathcal{V}^{j}\right\}$ so that $\mathcal{S}_{i}$ is the set of safety constraint indices where $u_{i}$ has an effect on the time derivative of the associated barrier function for some $x \in \mathcal{D}$. For the three vehicle example of Section IV] $\mathcal{S}_{1}=\{1,2\}, \mathcal{S}_{2}=\{1,3\}, \mathcal{S}_{3}=\{2,3\}$. The limited communication admissible control space for vehicle $i$ is then $\mathcal{K}_{i}(x)=\bigcap_{l \in \mathcal{S}_{i}} \mathcal{K}_{i}^{l}(x)$ and the overall limited communication admissible control space is

$$
\mathcal{K}(x)=\left\{u=\left[\begin{array}{lll}
u_{1}^{T} & \cdots & u_{k}^{T}
\end{array}\right]^{T} \in U: u_{i} \in \mathcal{K}_{i}(x) \forall i \in\{1, \ldots, k\}\right\} .
$$

Theorem 4. Given a dynamical system (1) and a set $C_{\cap} \subset \mathcal{D}_{\cap}$ defined in 25 for $q$ continuously differentiable functions $h^{j}$ defined in (27) with safety functions $\rho^{j}$ and evading maneuvers $\gamma^{j}$ where $k \in\{1, \ldots, q\}$, if $h^{j}$ is a ZCBF for $k \in\{1, \ldots, q\}$ and Assumption 1 holds then $\forall x \in \mathcal{D}_{\cap}, \mathcal{K}(x) \subseteq K_{\cap}(x)$. Further, $\mathcal{K}(x)$ is non-empty for all $x \in C_{\cap}$. If in addition, $\gamma^{s}$ maps to the interior of $U$ and for all $x \in \partial C_{\cap},\left\langle\left[L_{g} h^{j_{1}}(x)\right]_{i},\left[L_{g} h^{j_{2}}(x)\right]_{i}\right\rangle>0$ for $j=1, \ldots, q$ and $i=1, \ldots, k$ and $j_{1} \neq j_{2}$ and $j_{1}, j_{2} \in\{1, \ldots, q\}$, then there is an open set that is a superset of $C_{\cap}$ for which $\mathcal{K}(x)$ is non-empty for all $x$ in the open set.

Proof. For the first statement, assume $u \in \mathcal{K}(x)$ so that $u_{i} \in \mathcal{K}_{i}(x) \forall i \in\{1, \ldots, k\}$. This means that $A_{i} u_{i} \geq b_{i}$ so that, because $A$ is block diagonal, $A u \geq b$. Further, it means that for any constraint $j=1, \ldots, q$ and any $i \in \mathcal{V}^{j}$,

$0 \leq L_{f} h^{j, s}(x)+\left[L_{g} h^{j, s}(x)\right]_{i} u_{i}+\alpha\left(h^{j, s}(x)\right)+\left[L_{g} h^{j, s}(x)\right]_{\backslash i} \gamma_{\backslash i}^{s}(x)-\frac{\left|\mathcal{V}^{j}\right|-1}{\left|\mathcal{V}^{j}\right|}\left(L_{f} h^{j, s}(x)+L_{g} h^{j, s}(x) \gamma^{s}(x)+\alpha\left(h^{j, s}(x)\right)\right)$.

To simplify 34 , note that by definition, $\left[L_{g} h^{j, s}(x)\right]_{i}=0_{m_{i}}$ for $i \neq \mathcal{V}^{j}$ so that

$$
\begin{aligned}
\sum_{i \in \mathcal{V}^{j}}\left[L_{g} h^{j, s}(x)\right]_{i} u_{i} & =\sum_{i \in\{1, \ldots, k\}}\left[L_{g} h^{j, s}(x)\right]_{i} u_{i} \\
& =L_{g} h^{j, s}(x) u .
\end{aligned}
$$


Using $[35$ in the following then yields

$$
\begin{aligned}
\sum_{i \in \mathcal{V}^{j}}\left[L_{g} h^{j, s}(x)\right]_{\backslash i} \gamma_{\backslash i}^{s}(x) & =\sum_{i \in \mathcal{V}^{j}}\left(L_{g} h^{j, s}(x) \gamma^{s}(x)-\left[L_{g} h^{j, s}(x)\right]_{i} \gamma_{i}^{s}(x)\right) \\
& =\left|\mathcal{V}^{j}\right| L_{g} h^{j, s}(x) \gamma^{s}(x)-\sum_{i \in \mathcal{V}^{j}}\left[L_{g} h^{j, s}(x)\right]_{i} \gamma_{i}^{s}(x) \\
& =\left|\mathcal{V}^{j}\right| L_{g} h^{j, s}(x) \gamma^{s}(x)-L_{g} h^{j, s}(x) \gamma^{s}(x) \\
& =\left(\left|\mathcal{V}^{j}\right|-1\right) L_{g} h^{j, s}(x) \gamma^{s}(x) .
\end{aligned}
$$

Summing 34 , over $i \in \mathcal{V}^{j}$ and using 35 , and 36 yields

$$
\begin{aligned}
0 \leq & \left|\mathcal{V}^{j}\right| L_{f} h^{j, s}(x)+L_{g} h^{j, s}(x) u+\left|\mathcal{V}^{j}\right| \alpha\left(h^{j, s}(x)\right)+\left(\left|\mathcal{V}^{j}\right|-1\right) L_{g} h^{j, s}(x) \gamma^{s}(x) \\
& -\left(\left|\mathcal{V}^{j}\right|-1\right)\left(L_{f} h^{j, s}(x)+L_{g} h^{j, s}(x) \gamma^{s}(x)+\alpha\left(h^{j, s}(x)\right)\right) \\
= & L_{f} h^{j, s}(x)+L_{g} h^{j, s}(x) u+\alpha\left(h^{j, s}(x)\right) .
\end{aligned}
$$

Since this is true for all $j=1, \ldots, q, u \in K_{\cap}(x)$. Then $\mathcal{K}(x) \subseteq K_{\cap}(x)$ for all $x \in C_{\cap}$.

Consider now the second statement, namely that $\gamma^{s} \in \mathcal{K}(x)$. For $j=1, \ldots, q$, consider any $i \in \mathcal{V}^{j}$ and let $u_{i}=\gamma_{i}^{s}$.

Then

$$
\begin{aligned}
& L_{f} h^{j, s}(x)+\left[L_{g} h^{j, s}(x)\right]_{i} u_{i}+\alpha\left(h^{j, s}(x)\right)+\left[L_{g} h^{j, s}(x)\right]_{\backslash i} \gamma_{\backslash i}^{s}(x)-\frac{\left|\mathcal{V}^{j}\right|-1}{\left|\mathcal{V}^{j}\right|}\left(L_{f} h^{j, s}(x)+L_{g} h^{j, s}(x) \gamma^{s}(x)+\alpha\left(h^{j, s}(x)\right)\right) \\
& \quad=\frac{1}{\left|\mathcal{V}^{j}\right|}\left(L_{f} h^{j}(x)+L_{g} h^{j}(x) \gamma^{s}(x)+\alpha\left(h^{j}(x)\right)\right) \\
& \quad \geq 0
\end{aligned}
$$

The inequality is true because $x \in C_{\cap}$ implies $\alpha\left(h^{j, s}(x)\right) \geq 0$. See the proof for Theorem 2 for why $L_{f} h^{j, s}(x)+$ $L_{g} h^{j, s}(x) \gamma^{s}(x) \geq 0$. Then $\gamma_{i}^{s} \in \mathcal{K}_{i}^{j}$ for any $j=1, \ldots, q$ and $i \in \mathcal{V}^{j}$. Then $\gamma_{i}^{s} \in \mathcal{K}_{i}$. Then $\gamma^{s}(x) \in \mathcal{K}(x)$.

Finally, the last statement where $\mathcal{K}(x)$ is nonempty for all $x$ in an open set that is a superset of $\mathcal{C}_{\cap}$ follows similarly to the proof of Theorem 3

We now write a QP similar to (32) but without requiring knowledge of other agents' low level control values as 
follows:

$$
\begin{array}{ll}
u_{i}^{*}= & \min _{u_{i} \in \mathbb{R}^{m_{i}}} \frac{1}{2}\left\|u_{i}-\hat{u}_{i}\right\|^{2} \\
\text { s.t. } & A_{i} u_{i} \geq b_{i} \\
& L_{f} h^{j, s}(x)+\left[L_{g} h^{j, s}(x)\right]_{i} u_{i}+\alpha\left(h^{j, s}(x)\right)+\left[L_{g} h^{j, s}(x)\right]_{\backslash i} \gamma_{\backslash i}^{s}(x) \\
& \quad-\frac{\left|\mathcal{V}^{j}\right|-1}{\left|\mathcal{V}^{j}\right|}\left(L_{f} h^{j, s}(x)+L_{g} h^{j, s}(x) \gamma^{s}(x)+\alpha\left(h^{j, s}(x)\right)\right) \geq 0 \quad j \in \mathcal{S}_{i} .
\end{array}
$$

We note that the solution from the centralized QP 32 may be different than the solution from the limited communication QPs (37) because $\mathcal{K}(x)$ may be a strict subset of $K_{\cap}(x)$. To see this, let $k=2, q=1, L_{f} h(x)=0$, $\alpha(h(x))=0, m_{1}=m_{2}=1,\left[L_{g} h(x)\right]_{2} \gamma_{2}^{s}(x)=-1$, and $\left[L_{g} h(x)\right]_{1} \gamma_{1}^{s}(x)=1$. Then the barrier function constraint in (37) becomes $\left[L_{g} h(x)\right]_{1} u_{1} \geq 1$, while the barrier function constraint in 32 becomes $L_{g} h(x) u \geq 0$. Since $u_{1}=0$ is feasible for the latter but not the former equation, we do not have that $\mathcal{K}(x)=K_{\cap}(x)$. Because $\mathcal{K}(x) \subset K_{\cap}(x)$, it may be that the total cost of each vehicle calculating 37) is higher than the centralized calculation 32. In other words, the calculated safe control may not be as close to the nominal control signal in a least squares sense when using (37) as opposed to (32). Nevertheless, in either case of (32) or (37), a solution exists to the corresponding QP such that $u \in K_{\cap}$.

Another difference between the limited communication (37) and the centralized (32) QPs is how the size of the optimization variable and number of constraints vary with the number of vehicles $k$. In the centralized approach 32 the size of the optimization variable grows linearly with $k$ while the number of constraints grows quadratically. On the other hand, in the limited communication QP 37 , the size of the optimization variable and number of constraints are constant and linear, respectively.

\section{SIMULATION}

In this section we repeat the scenario discussed in Section III.D but consider $k=20$ vehicles. For the scenario where $h$ is constructed from $\gamma_{\text {turn }}$, we use $\left[\begin{array}{lllllll}v & \omega & 0 & v & \omega & 0\end{array}\right]^{T}$ where $v=0.9 v_{\text {min }}+0.1 v_{\text {max }}$ and $\omega=0.9 \omega_{\text {max }}$. For the scenario where $h$ is constructed from $\gamma_{\text {straight }}$, we let $\gamma^{i}=\left[\begin{array}{ccc}(1+0.01 i) v & 0 & 0\end{array}\right]^{T}$ so that each vehicle uses a different translational velocity as is required to ensure differentiability of $h$ (see Section III.C). Note that this does not violate the shared evading maneuver assumption because $\gamma^{s}=\left[\begin{array}{lll}\left(\gamma^{1}\right)^{T} & \cdots & \left(\gamma^{k}\right)^{T}\end{array}\right]^{T}$. Additionally, we let $\psi=0$ and $\psi=25^{\circ}$ in the scenario where $h$ is constructed from $\gamma_{t u r n}$ and $\gamma_{\text {straight }}$, respectively. Offsetting the initial orientation $25^{\circ}$ from pointing at the origin is required so that the vehicles can start in the safe set when using $\gamma_{\text {straight }}$. A video of the resulting behavior is available in [45]. Quantitative results for both scenarios are shown in Figure 6 which shows similar outputs to the results for the two vehicle simulation shown in Figure 2 Small random perturbations, e.g. adding 
a uniformly sampled angle within $\pm 5^{\circ}$ to the initial heading, does not appear to change qualitative results. We also compare the approach of this paper to a navigation function from [9] in Figure 6. Note that when using a navigation function the vehicles begin the evasive maneuver earlier than when the collision avoidance algorithm is based on a barrier function constructed from $\gamma_{t u r n}$. Using a less aggressive $\alpha$ function, such as a linear function with a small coefficient instead of a cubic function, may have caused the behavior from the barrier function override to similarly override earlier. A comparison of potential and barrier functions can also be found in [46]. Also note that the pairwise distance between all vehicles are kept above the minimum safety distance $D_{s}$ while satisfying actuator constraints.

\section{CONCLUSION}

In this paper we have examined method for ensuring a system with constrained inputs can be safe for all future times. The main result is a general method for constructing a barrier function given a safety constraint, system dynamics with actuator limits, and an evasive function specified by a safety engineer. We then apply this method to show how collision avoidance for two UAVs can be ensured for all future times. The result is then extended to the case of collision avoidance for arbitrarily many UAVs by considering how to ensure that arbitrarily many safety objectives can be satisfied simultaneously. In the case of arbitrarily many UAVs, network constraints may limit the message throughput so we provide a reformulation of the algorithm that requires less message passing while still ensuring that vehicles will stay safe. The final result is demonstrated in a simulation of 20 UAVs where the vehicles are on a collision course. However, due to the role of the barrier function in ensuring safety, all twenty vehicles nevertheless maintain safe distances from each other and then able to reach their assigned waypoints.

\section{Appendix}

\section{An Analysis of The Role of $\delta$ in The Continuous Differentiability of $h_{t u r n}$}

Note that 16 is not necessarily differentiable when $A_{2}=0$ since $A_{2}$ results from a square root performed in phasor addition. Thus, in this section, we consider how to ensure $A_{2}$ is continuously differentiable to ensure $h$ in (16) is continuously differentiable. Consider $(16)$ in phasor form

$$
\begin{aligned}
A_{1}-D_{s}^{2}+A_{2} e^{j \Theta}= & A_{1}-D_{s}^{2}+\sigma A_{3} e^{j\left(\theta_{1,0}-\pi / 2\right)}+A_{3} e^{j\left(\theta_{2,0}+\pi / 2\right)}+\delta e^{j\left(\theta_{1,0}-\pi / 2\right)} \\
& +\sigma A_{4} e^{j\left(\theta_{1,0}-\pi\right)}+A_{4} e^{j \theta_{2,0}}+\delta e^{j\left(\theta_{1,0}-\pi\right)} \\
= & A_{1}-D_{s}^{2}+A_{5} e^{j \Theta_{5}}+A_{6} e^{j \Theta_{6}}
\end{aligned}
$$

where $A_{3}=2 \Delta b_{0} r, A_{4}=2 \Delta c_{0} r, A_{5} e^{j \Theta_{5}}=\sigma A_{3} e^{j\left(\theta_{1,0}-\pi / 2\right)}+A_{3} e^{j\left(\theta_{2,0}+\pi / 2\right)}+\delta e^{j\left(\theta_{1,0}-\pi / 2\right)}$, and $A_{6} e^{j \Theta_{6}}=\sigma A_{4} e^{j\left(\theta_{1,0}-\pi\right)}+$ $A_{4} e^{j \theta_{2,0}}+\delta e^{j\left(\theta_{1,0}-\pi\right)}$. Notice that $\Theta_{5}-\Theta_{6}=\pi / 2$. In other words, $A_{2}$ is zero only when both $A_{5}$ and $A_{6}$ are zero. For 


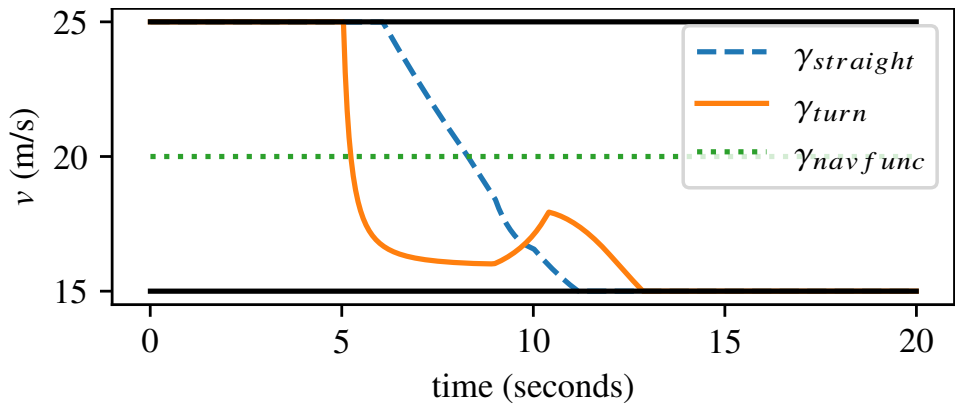

(a)

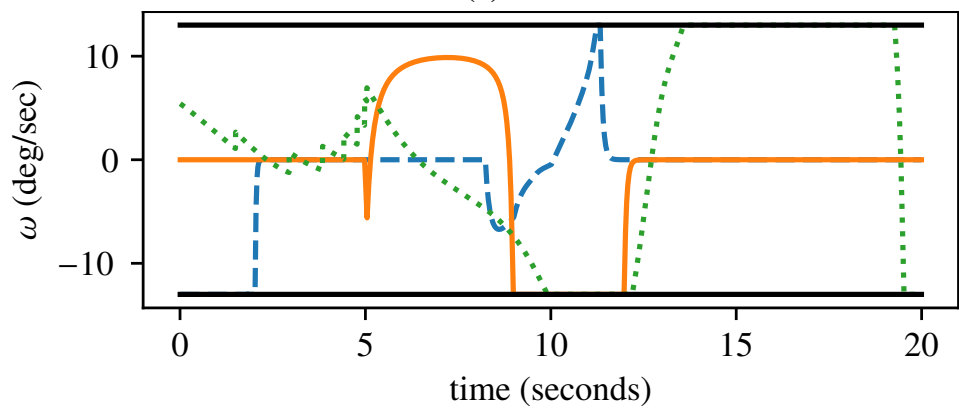

(b)

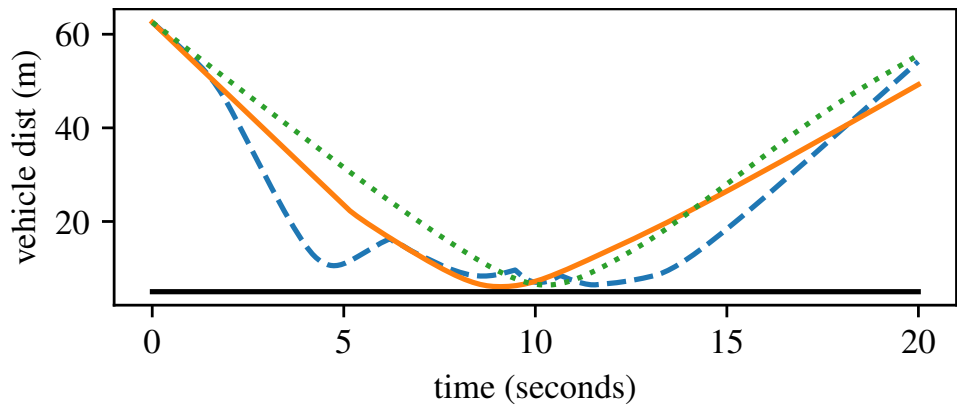

(c)

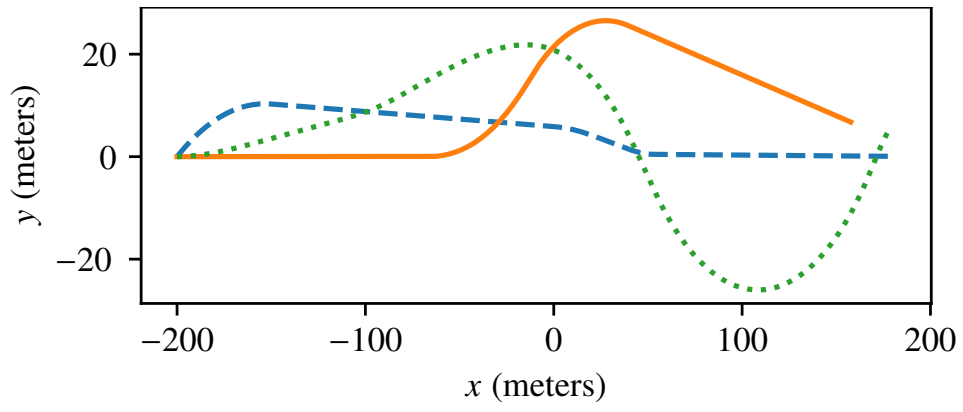

(d)

Fig. 620 vehicle scenario statistics. (a) vehicle 1 velocity, (b) vehicle 1 turn rate, (c) intervehicle distance, (d) vehicle 1 path. 
$\delta=0, A_{5}$ and $A_{6}$ are both zero on the set $Z_{1} \subseteq \mathcal{D}$ where $\theta_{1,0}=\theta_{2,0}$ or $\theta_{1,0}=\theta_{2,0}+\pi$. Although $Z_{1}$ is a zero measure set, we note that for $\delta>0$ that $A_{2}$ is zero on a set $Z_{2} \subset Z_{1}$ where $Z_{2}$ is the restriction of $Z_{1}$ to a specific set of positions which we now specify.

Case 1. Vehicles Start in Opposite Directions. Suppose $\theta_{1,0}=\theta_{2,0}+\pi$. Then $A_{5}=0$ when $\delta=-(1+\sigma) A_{3}=$ $-2(1+\sigma) \Delta b_{0} r$. Similarly, $A_{6}=0$ when $\delta=-(1+\sigma) A_{4}=-2(1+\sigma) \Delta c_{0} r$. Suppose $\delta$ is fixed. Then $A_{2}=0$ when $-\frac{\delta}{2(1+\sigma) r}=\Delta b_{0}=p_{1, x_{0}}-p_{2, x_{0}}+r(1+\sigma) \sin \theta_{2,0}$ and $-\frac{\delta}{2(1+\sigma) r}=\Delta c_{0}=p_{1, y_{0}}-p_{2, y_{0}}-r(1+\sigma) \cos \theta_{2,0}$.

Case 2. Vehicles Start in the Same Direction. Suppose $\theta_{1,0}=\theta_{2,0}$. Then $A_{5}=0$ when $\delta=(1-\sigma) A_{3}$. Similarly, $A_{6}=0$ when $\delta=(1-\sigma) A_{4}$. For $\sigma=1$, let $\delta>0$ to ensure $A_{5}$ and $A_{6}$ are not simultaneously 0 . For $0<\sigma<1$, a similar analysis to the previous case implies $A_{2}=0$ when when $-\frac{\delta}{2(1-\sigma) r}=\Delta b_{0}=p_{1, x_{0}}+p_{2, x_{0}}-r(1-\sigma) \sin \theta_{2,0}$ and $-\frac{\delta}{2(1+\sigma) r}=\Delta c_{0}=p_{1, y_{0}}-p_{2, y_{0}}-r(1+\sigma) \cos \theta_{2,0}$

\section{An Analysis of the Continuous Differentiability of $h_{\text {straight }}$}

From 19 we expand terms to get

$$
h(x)=\inf _{\tau \in[0, \infty)} c(x)+b(x) \tau+a(x) \tau^{2}
$$

where $c(x)=\Delta x^{2}+\Delta y^{2}+\Delta z^{2}-D_{s}^{2}, b(x)=2(\Delta x \Delta C+\Delta y \Delta S), a(x)=\Delta C^{2}+\Delta S^{2}, \Delta x=p_{1, x_{0}}-p_{2, x_{0}}, \Delta y=p_{1, y_{0}}-p_{2, y_{0}}$, $\Delta z=p_{1, z_{0}}-p_{2, z_{0}}, \Delta C=v_{1} \cos \theta_{1}-v_{2} \cos \theta_{2}, \Delta S=v_{1} \sin \theta_{1}-v_{2} \sin \theta_{2}$. We also note that $a(x)>0$ since

$$
\begin{aligned}
a(x) & =\left(v_{1} \cos \theta_{1}-v_{2} \cos \theta_{2}\right)^{2}+\left(v_{1} \sin \theta_{1}-v_{2} \sin \theta_{2}\right)^{2} \\
& =v_{1}^{2}+v_{2}^{2}-2 v_{1} v_{2} \cos \left(\theta_{1}-\theta_{2}\right) \\
& =v_{1}^{2}+v_{2}^{2}-2 v_{1} v_{2}+2 v_{1} v_{2}-2 v_{1} v_{2} \cos \left(\theta_{1}-\theta_{2}\right) \\
& =\left(v_{1}-v_{2}\right)^{2}+2 v_{1} v_{2}\left(1-\cos \left(\theta_{1}-\theta_{2}\right)\right) \\
& >0
\end{aligned}
$$

since $v_{1} \neq v_{2}$ and $v_{1}$ and $v_{2}$ are positive. Then $\tau_{\min }(x)=-b(x) / 2 a(x)$ is well defined. Then $h$ has a minimum at $\tau_{\text {nonneg, } \min }=\max \left(0, \tau_{\min }(x)\right)$.

For $\tau_{\text {nonneg, } \min }(x)>0, h$ is continuously differentiable because $c, b, \tau_{\min }$, and $a$ are continuously differentiable. Consider now when $\tau_{\text {nonneg, } \min }(x)=0$. We verify that $\frac{\partial h(x)}{\partial x}=\frac{\partial c(x)}{\partial x}$ for either the case of $\tau_{\text {min }}=0$ or $\tau_{\text {min }}=$ $-b(x) / 2 a(x)$. In the first case, $h(x)=c(x)$ and $\frac{\partial h(x)}{\partial x}=\frac{\partial c(x)}{\partial x}$. In the second case, $h(x)=c(x)+b(x) \tau_{\min }+a(x) \tau_{\text {min }}^{2}$ 
and

$$
\begin{aligned}
\frac{\partial h(x)}{\partial x} & =\frac{\partial c(x)}{\partial x}+\frac{\partial b(x)}{\partial x} \tau_{\min }(x)+b(x) \frac{\partial \tau_{\min }(x)}{\partial x}+\frac{\partial a(x)}{\partial x} \tau_{\min }(x)+2 a(x) \tau_{\min } \frac{\partial \tau_{\min }(x)}{\partial x} \\
& =\frac{\partial c(x)}{\partial x}
\end{aligned}
$$

because in this case $b(x)$ and $\tau_{\min }(x)$ are 0.

\section{Funding Sources}

The work of Eric Squires was supported by the University System of Georgia's Tuition Assistance Program. The work by Magnus Egerstedt and Pietro Pierpaoli was supported by Grant No. ARL DCIST CRA W911NF-17-2-0181 by the US Army Research Lab. The work of Samuel Coogan and Rohit Konda was supported by the Air Force Office of Scientific Research under grant number FA9550-19-1-0015.

\section{References}

[1] Prevot, T., Rios, J., Kopardekar, P., Robinson III, J. E., Johnson, M., and Jung, J., "UAS traffic management (UTM) concept of operations to safely enable low altitude flight operations," 16th AIAA Aviation Technology, Integration, and Operations Conference, 2016, p. 3292. https://doi.org/10.2514/6.2016-3292

[2] Temizer, S., Kochenderfer, M., Kaelbling, L., Lozano-Pérez, T., and Kuchar, J., "Collision avoidance for unmanned aircraft using Markov decision processes," AIAA guidance, navigation, and control conference, 2010, p. 8040. https://doi.org/10.2514/6.20108040

[3] Wolf, T. B., and Kochenderfer, M. J., "Aircraft collision avoidance using Monte Carlo real-time belief space search," Journal of Intelligent \& Robotic Systems, Vol. 64, No. 2, 2011, pp. 277-298. https://doi.org/10.1007/s10846-010-9532-6

[4] Fox, D., Burgard, W., and Thrun, S., “The dynamic window approach to collision avoidance," IEEE Robotics \& Automation Magazine, Vol. 4, No. 1, 1997, pp. 23-33. https://doi.org/10.1109/100.580977

[5] Seder, M., and Petrovic, I., "Dynamic window based approach to mobile robot motion control in the presence of moving obstacles," Robotics and Automation, 2007 IEEE International Conference on, IEEE, 2007, pp. 1986-1991. https://doi.org/10. 1109/ROBOT.2007.363613.

[6] Lalish, E., Morgansen, K. A., and Tsukamaki, T., "Decentralized reactive collision avoidance for multiple unicycle-type vehicles," American Control Conference, 2008, IEEE, 2008, pp. 5055-5061. https://doi.org/10.1109/ACC.2008.4587295.

[7] Mastellone, S., Stipanović, D. M., Graunke, C. R., Intlekofer, K. A., and Spong, M. W., "Formation control and collision avoidance for multi-agent non-holonomic systems: Theory and experiments," The International Journal of Robotics Research, Vol. 27, No. 1, 2008, pp. 107-126. https://doi.org/10.1177/0278364907084441 
[8] Rodriguez-Seda, E. J., "Decentralized trajectory tracking with collision avoidance control for teams of unmanned vehicles with constant speed," American Control Conference (ACC), 2014, IEEE, 2014, pp. 1216-1223. https://doi.org/10.1109/ACC.2014. 6859184

[9] Panyakeow, P., and Mesbahi, M., "Decentralized deconfliction algorithms for unicycle UAVs," American Control Conference (ACC), 2010, IEEE, 2010, pp. 794-799. https://doi.org/10.1109/ACC.2010.5530943.

[10] Di, B., Zhou, R., and Duan, H., "Potential field based receding horizon motion planning for centrality-aware multiple UAV cooperative surveillance," Aerospace Science and Technology, Vol. 46, 2015, pp. 386-397. https://doi.org/10.1016/j.ast.2015. 08.006

[11] Defoort, M., Kokosy, A., Floquet, T., Perruquetti, W., and Palos, J., “Motion planning for cooperative unicycle-type mobile robots with limited sensing ranges: A distributed receding horizon approach," Robotics and autonomous systems, Vol. 57, No. 11, 2009, pp. 1094-1106. https://doi.org/10.1016/j.robot.2009.07.004

[12] Shin, J., and Kim, H. J., "Nonlinear model predictive formation flight," IEEE Transactions on Systems, Man, and Cybernetics-Part A: Systems and Humans, Vol. 39, No. 5, 2009, pp. 1116-1125. https://doi.org/10.1109/TSMCA.2009.2021935

[13] Tomlin, C., Pappas, G. J., and Sastry, S., "Conflict resolution for air traffic management: A study in multiagent hybrid systems," IEEE Transactions on automatic control, Vol. 43, No. 4, 1998, pp. 509-521. https://doi.org/10.1109/9.664154

[14] Lai, C.-K., Lone, M., Thomas, P., Whidborne, J., and Cooke, A., "On-board trajectory generation for collision avoidance in unmanned aerial vehicles," Aerospace Conference, 2011 IEEE, IEEE, 2011, pp. 1-14. https://doi.org/10.1109/AERO.2011. 5747526

[15] Lin, Y., and Saripalli, S., "Path planning using 3D dubins curve for unmanned aerial vehicles," Unmanned Aircraft Systems (ICUAS), 2014 International Conference on, IEEE, 2014, pp. 296-304. https://doi.org/10.1109/ICUAS.2014.6842268

[16] Lin, Y., and Saripalli, S., "Collision avoidance for UAVs using reachable sets," Unmanned Aircraft Systems (ICUAS), 2015 International Conference on, IEEE, 2015, pp. 226-235. https://doi.org/10.1109/ICUAS.2015.7152295

[17] Kolmanovsky, I., Garone, E., and Di Cairano, S., "Reference and command governors: A tutorial on their theory and automotive applications," American Control Conference (ACC), 2014, IEEE, 2014, pp. 226-241. https://doi.org/10.1109/ACC.2014. 6859176

[18] Tedesco, F., Raimondo, D. M., and Casavola, A., "Collision avoidance command governor for multi-vehicle unmanned systems," International Journal of Robust and Nonlinear Control, Vol. 24, No. 16, 2014, pp. 2309-2330. https://doi.org/10.1002/rnc.2989

[19] Althoff, D., Althoff, M., and Scherer, S., "Online safety verification of trajectories for unmanned flight with offline computed robust invariant sets," 2015 IEEE/RSJ International Conference on Intelligent Robots and Systems (IROS), IEEE, 2015, pp. 3470-3477. https://doi.org/10.1109/IROS.2015.7353861. 
[20] Pallottino, L., Scordio, V. G., Bicchi, A., and Frazzoli, E., "Decentralized cooperative policy for conflict resolution in multivehicle systems," IEEE Transactions on Robotics, Vol. 23, No. 6, 2007, pp. 1170-1183. https://doi.org/10.1109/TRO.2007.909810

[21] Krontiris, A., and Bekris, K. E., "Using minimal communication to improve decentralized conflict resolution for non-holonomic vehicles," Intelligent Robots and Systems (IROS), 2011 IEEE/RSJ International Conference on, IEEE, 2011, pp. 3235-3240. https://doi.org/10.1109/IROS.2011.6095175

[22] Prajna, S., "Barrier certificates for nonlinear model validation," Automatica, Vol. 42, No. 1, 2006, pp. 117-126. URL 10.1016/j.automatica.2005.08.007

[23] Ames, A. D., Xu, X., Grizzle, J. W., and Tabuada, P., "Control barrier function based quadratic programs for safety critical systems," IEEE Transactions on Automatic Control, Vol. 62, No. 8, 2017, pp. 3861-3876. https://doi.org/10.1109/TAC.2016.2638961

[24] Borrmann, U., Wang, L., Ames, A. D., and Egerstedt, M., "Control barrier certificates for safe swarm behavior," IFACPapersOnLine, Vol. 48, No. 27, 2015, pp. 68-73. https://doi.org/10.1016/j.ifacol.2015.11.154.

[25] Wang, L., Ames, A. D., and Egerstedt, M., "Safe certificate-based maneuvers for teams of quadrotors using differential flatness," 2017 IEEE International Conference on Robotics and Automation (ICRA), IEEE, 2017, pp. 3293-3298. https: //doi.org/10.1109/ICRA.2017.7989375

[26] Nguyen, Q., and Sreenath, K., "Safety-critical control for dynamical bipedal walking with precise footstep placement," IFAC-PapersOnLine, Vol. 48, No. 27, 2015, pp. 147-154. https://doi.org/10.1016/j.ifacol.2015.11.167

[27] Hsu, S.-C., Xu, X., and Ames, A. D., "Control barrier function based quadratic programs with application to bipedal robotic walking," American Control Conference (ACC), 2015, IEEE, 2015, pp. 4542-4548. https://doi.org/10.1109/ACC.2015.7172044

[28] Xu, X., Grizzle, J. W., Tabuada, P., and Ames, A. D., "Correctness guarantees for the composition of lane keeping and adaptive cruise control," IEEE Transactions on Automation Science and Engineering, 2017. https://doi.org/10.1109/TASE.2017.2760863

[29] Xu, X., Tabuada, P., Grizzle, J. W., and Ames, A. D., "Robustness of control barrier functions for safety critical control," IFAC-PapersOnLine, Vol. 48, No. 27, 2015, pp. 54-61. https://doi.org/10.1016/j.ifacol.2015.11.152

[30] Xu, X., Waters, T., Pickem, D., Glotfelter, P., Egerstedt, M., Tabuada, P., Grizzle, J. W., and Ames, A. D., "Realizing simultaneous lane keeping and adaptive speed regulation on accessible mobile robot testbeds," Control Technology and Applications (CCTA), 2017 IEEE Conference on, IEEE, 2017, pp. 1769-1775. https://doi.org/10.1109/CCTA.2017.8062713.

[31] Wang, L., Ames, A. D., and Egerstedt, M., "Multi-objective compositions for collision-free connectivity maintenance in teams of mobile robots," Decision and Control (CDC), 2016 IEEE 55th Conference on, IEEE, 2016, pp. 2659-2664. https://doi.org/10.1109/CDC.2016.7798663

[32] Prajna, S., and Jadbabaie, A., "Safety verification of hybrid systems using barrier certificates," HSCC, Vol. 2993, Springer, 2004, pp. 477-492. https://doi.org/10.1007/978-3-540-24743-2_32 
[33] Wang, L., Han, D., and Egerstedt, M., "Permissive barrier certificates for safe stabilization using sum-of-squares," 2018 Annual American Control Conference (ACC), IEEE, 2018, pp. 585-590. https://doi.org/10.23919/ACC.2018.8431617

[34] Parrilo, P. A., "Semidefinite programming relaxations for semialgebraic problems," Mathematical programming, Vol. 96, No. 2, 2003, pp. 293-320. https://doi.org/10.1007/s10107-003-0387-5

[35] Nguyen, Q., and Sreenath, K., "Exponential control barrier functions for enforcing high relative-degree safety-critical constraints," American Control Conference (ACC), 2016, IEEE, 2016, pp. 322-328. https://doi.org/10.1109/ACC.2016.7524935

[36] Xu, X., "Constrained control of input-output linearizable systems using control sharing barrier functions," Automatica, Vol. 87, 2018, pp. 195-201. https://doi.org/10.1016/j.automatica.2017.10.005

[37] Gurriet, T., Mote, M., Ames, A. D., and Feron, E., "An online approach to active set invariance," 2018 IEEE Conference on Decision and Control (CDC), IEEE, 2018, pp. 3592-3599. https://doi.org/10.1109/CDC.2018.8619139.

[38] Glotfelter, P., Cortés, J., and Egerstedt, M., “Nonsmooth Barrier Functions With Applications to Multi-Robot Systems,” IEEE control systems letters, Vol. 1, No. 2, 2017, pp. 310-315. https://doi.org/10.1109/LCSYS.2017.2710943

[39] (C2018 IEEE, "Reprinted, with permission, from Eric Squires, Pietro Pierpaoli, and Magnus Egerstedt, "Constructive Barrier Certificates With Applications To Fixed-Wing Aircraft Collision Avoidance", https://doi.org/10.1109/CCTA.2018.8511342|", Aug 2018.

[40] DeMarco, K., Squires, E., Day, M., and Pippin, C., "Simulating collaborative robots in a massive multi-agent game environment (SCRIMMAGE)," Distributed Autonomous Robotic Systems, Springer, 2019, pp. 283-297. https://doi.org/10.1007/978-3-03005816-6_20

[41] Olfati-Saber, R., "Near-identity diffeomorphisms and exponential/spl epsi/-tracking and/spl epsi/-stabilization of first-order nonholonomic SE (2) vehicles," American Control Conference, 2002. Proceedings of the 2002, Vol. 6, IEEE, 2002, pp. 4690-4695. https://doi.org/10.1109/ACC.2002.1025398

[42] Clancy, L. J., Aerodynamics, Halsted Press, 1975.

[43] Stellato, B., Banjac, G., Goulart, P., Bemporad, A., and Boyd, S., "OSQP: An Operator Splitting Solver for Quadratic Programs," ArXiv e-prints, 2017.

[44] Blanchini, F., and Miani, S., Set-theoretic methods in control, Springer, 2008.

[45] Squires, E., "Composition of Safety Constraints For Fixed-Wing Collision Avoidance Amidst Limited Communications," https://youtu.be/5y015taoJw4. 2020. Accessed: 2020-02-10.

[46] Singletary, A., Klingebiel, K., Bourne, J., Browning, A., Tokumaru, P., and Ames, A., "Comparative analysis of control barrier functions and artificial potential fields for obstacle avoidance," arXiv preprint arXiv:2010.09819, 2020. 